\title{
Impact of high energy high intensity proton beams on targets: Case studies for Super Proton Synchrotron and Large Hadron Collider
}

\author{
N. A. Tahir, ${ }^{1}$ J. Blanco Sancho, ${ }^{2}$ A. Shutov, ${ }^{3}$ R. Schmidt, ${ }^{4}$ and A. R. Piriz ${ }^{5}$ \\ ${ }^{1}$ GSI Helmholzzentrum für Schwerionenforschung, Planckstraße 1, 64291 Darmstadt, Germany \\ ${ }^{2}$ CERN-AB, 1211 Geneva 23, Switzerland, and EPFL Lausanne, Switzerland \\ ${ }^{3}$ Institute of Problems of Chemical Physics, Russian Academy of Sciences, Institutskii Prospekt 18, 142432 Chernogolovka, Russia \\ ${ }^{4} C E R N-A B, 1211$ Geneva 23, Switzerland \\ ${ }^{5}$ E.T.S.I. Industriales, Universidad de Castilla-La Mancha, 13071 Ciudad Real, Spain
}

(Received 18 November 2011; published 8 May 2012)

\begin{abstract}
The Large Hadron Collider (LHC) is designed to collide two proton beams with unprecedented particle energy of $7 \mathrm{TeV}$. Each beam comprises 2808 bunches and the separation between two neighboring bunches is $25 \mathrm{~ns}$. The energy stored in each beam is $362 \mathrm{MJ}$, sufficient to melt $500 \mathrm{~kg}$ copper. Safety of operation is very important when working with such powerful beams. An accidental release of even a very small fraction of the beam energy can result in severe damage to the equipment. The machine protection system is essential to handle all types of possible accidental hazards; however, it is important to know about possible consequences of failures. One of the critical failure scenarios is when the entire beam is lost at a single point. In this paper we present detailed numerical simulations of the full impact of one LHC beam on a cylindrical solid carbon target. First, the energy deposition by the protons is calculated with the FLUKA code and this energy deposition is used in the BIG2 code to study the corresponding thermodynamic and the hydrodynamic response of the target that leads to a reduction in the density. The modified density distribution is used in FLUKA to calculate new energy loss distribution and the two codes are thus run iteratively. A suitable iteration step is considered to be the time interval during which the target density along the axis decreases by $15 \%-20 \%$. Our simulations suggest that the full LHC proton beam penetrates up to $25 \mathrm{~m}$ in solid carbon whereas the range of the shower from a single proton in solid carbon is just about $3 \mathrm{~m}$ (hydrodynamic tunneling effect). It is planned to perform experiments at the experimental facility HiRadMat (High Radiation Materials) at CERN using the proton beam from the Super Proton Synchrotron (SPS), to compare experimental results with the theoretical predictions. Therefore simulations of the response of a solid copper cylindrical target hit by the SPS beam were performed. The particle energy in the SPS beam is $440 \mathrm{GeV}$ while it has the same bunch structure as the LHC beam, except that it has only up to 288 bunches. Beam focal spot sizes of $\sigma=0.1,0.2$, and $0.5 \mathrm{~mm}$ have been considered. The phenomenon of significant hydrodynamic tunneling due to the hydrodynamic effects is also expected for the experiments.
\end{abstract}

DOI: 10.1103/PhysRevSTAB.15.051003

PACS numbers: 29.27.- a

\section{INTRODUCTION}

Extensive simulation studies of the full impact of the ultrarelativistic proton beam generated by the Large Hadron Collider (LHC) on solid targets of different materials of interest have been carried out over the past years. In our first study, we simulated the response of a solid copper cylindrical target that was facially irradiated by one LHC beam [1] along the axis. These simulations were done using a two-dimensional hydrodynamic computer code, BIG2 [2]. The energy deposition of the $7 \mathrm{TeV} / c$ protons in copper was calculated with the FLUKA code $[3,4]$ assuming solid target density. This data was used as input to the

Published by the American Physical Society under the terms of the Creative Commons Attribution 3.0 License. Further distribution of this work must maintain attribution to the author(s) and the published article's title, journal citation, and DOI.
BIG2 code. In these simulations we studied the target behavior along the cross section at a fixed point on the axis $(L=16 \mathrm{~cm})$, where the maximum energy deposition occurred. This study showed that the high pressure produced in the deposition region after energy deposition by only 100 proton bunches generated a radially outgoing shock wave that led to a substantial reduction in the density at the center. In practice, the protons in subsequent bunches will penetrate much deeper into the target. However, due to the limitations of the model used in those calculations, this effect could not be simulated. The penetration depth of $7 \mathrm{TeV} / c$ protons in solid copper was calculated using analytic estimates based on these simulations. It was predicted that the LHC protons can penetrate between $10-40 \mathrm{~m}$ in solid copper. A very interesting and important outcome of this study was that a significant part of the target was converted into a sample of high energy density (HED) matter. This work therefore suggested that the LHC 
can also be used as a tool to study HED physics, which could be an additional, very important application of the LHC [5].

A major improvement in the work was made when we carried out two-dimensional hydrodynamic simulations of a cylindrical copper target in the $\mathrm{r}-\mathrm{Z}$ geometry. Although we still used the same proton energy deposition data as in the previous case (considering solid target density), the reduction in the energy deposition of the subsequently delivered bunches in the already heated low-density region was simulated by normalizing the solid-density energy deposition with the line density along the axis. This approximation allowed us to simulate the range lengthening phenomenon (hydrodynamic tunneling effect) in the simulations. This analytic model provided a very good insight into this problem. These simulations suggested that the LHC beam will penetrate up to $35 \mathrm{~m}$ in solid copper due to the hydrodynamic tunneling effect [6], which was within the range of our previous prediction.

Recently, we have accomplished major advancement in this work by simulating the hydrodynamic and the thermodynamic response of a solid carbon cylindrical target irradiated by the full LHC beam by using the FLUKA and the BIG2 codes, iteratively. The iteration interval is considered to be the time during which the target density decreases around the target axis by 15\%-20\% due to the hydrodynamic effects. These simulations predict that the LHC protons and the shower will penetrate up to $25 \mathrm{~m}$ in solid carbon. It is interesting to note that excluding the hydrodynamic effects, LHC protons and the developed shower penetrate in solid carbon only up to around $3 \mathrm{~m}$.

We note that the experimental verification of the numerical simulations is very important from the machine protection point of view [7-9]. However, this is not possible with the LHC beam. For this purpose, experiments will be performed at an experimental facility named HiRadMat (High Radiation Materials) that has been constructed at CERN to carry out fixed target experiments using the Super Proton Synchrotron (SPS) proton beam with a particle energy of $440 \mathrm{GeV}$. To assist designing of suitable experiments, we have carried out extensive numerical simulations of heating of solid copper cylinders using the SPS beam and the results are presented in this paper. It is interesting to note that the hydrodynamic tunneling effect is also clearly observed in the case of these simulations. Confirmation of the existence of this very important phenomenon in the HiRadMat experiments will validate our simulations for the LHC beam.

In Sec. II we present the LHC, its nominal beam parameters, and the machine protection systems. In Sec. III we describe the SPS parameters and the HiRadMat facility while an overview of the previous calculations is given in Sec. IV. Numerical simulations of the full impact of the LHC beam on a solid carbon cylinder are given in Sec. V. Similar calculations of the interaction of the SPS beam with a solid copper cylinder are presented in Sec. VI. Conclusions drawn from this work are noted in Sec. VII.

\section{LARGE HADRON COLLIDER AT CERN}

The motivation to construct the Large Hadron Collider (LHC) [10] at CERN comes from fundamental questions of particle physics. The LHC has been installed in a tunnel with a circumference of $26.8 \mathrm{~km}$ that was previously used for the Large Electron Positron Collider (LEP). Two counterrotating proton beams circulate in separate beam pipes and will finally be accelerated to particle energies of $7 \mathrm{TeV}$. The protons in the two beams will then be made to collide at a center of mass energy of $14 \mathrm{TeV}$.

In order to achieve the required collision rate, each beam consists of a bunch train with each bunch consisting of $1.15 \times 10^{11}$ protons. The design parameters assume a total number of bunches in each beam is 2808 and the total number of protons per beam is about $3 \times 10^{14}$. The bunch length is $0.5 \mathrm{~ns}$ and two neighboring bunches are separated by $25 \mathrm{~ns}$ while the intensity distribution in radial direction is Gaussian with a standard deviation, $\sigma=0.2 \mathrm{~mm}$. In the center of the physics detectors the beam will be focused to a much smaller size, down to $\sigma=20 \mu \mathrm{m}$. The total duration of the beam is of the order of $89 \mu$ s since there are gaps between the bunch trains required for injection and extraction.

The LHC enters into a new domain. In particular, the energy stored in each beam is larger by about 2 orders of magnitude with respect to other hadron machines, such as ISR, SPS, HERA, TEVATRON, and SNS as shown in Fig. 1. Electron machines such as LEP operate with less stored energy. Handling beam with such huge energy is a significant challenge. Machine protection becomes vital, in particular in the presence of superconducting magnets that quench in case of beam losses in the order of a few $\mathrm{mJ}$.

The beam is prepared at the CERN injector complex that comprises several accelerators, linacs, booster, Proton Synchrotron, and finally the Super Proton Synchrotron

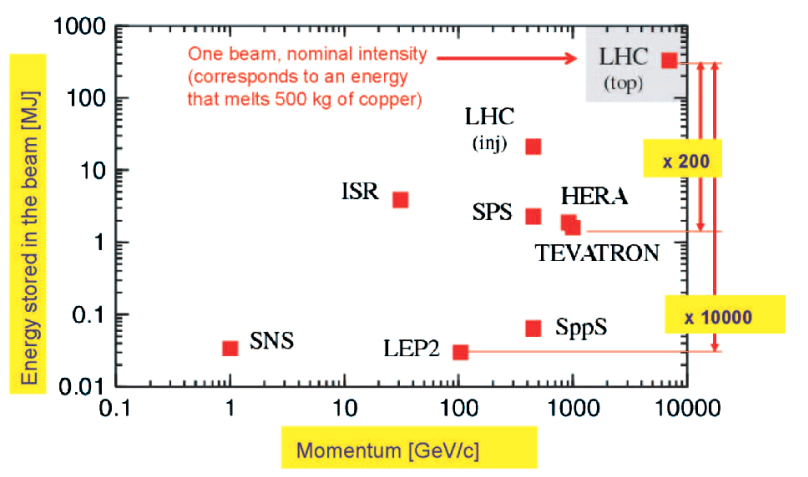

FIG. 1. Energy stored in the beams as a function of the particle momentum for different accelerators: CERN-ISR [36], CERNLEP2 [36], DESY-HERA [37], FERMILAB-TEVATRON [38], ORNL-SNS [39], and CERN-LHC. 


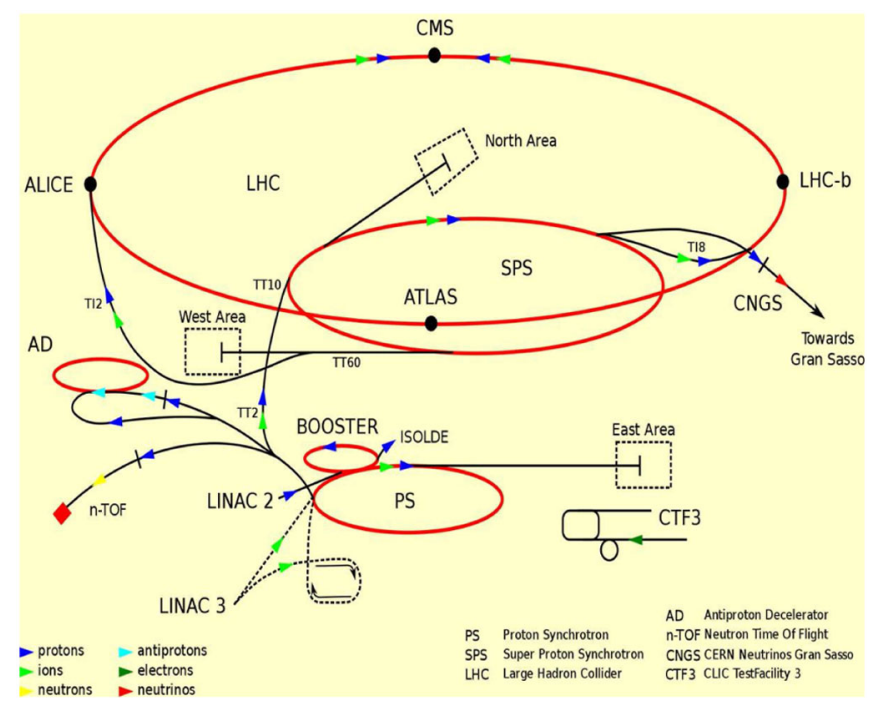

FIG. 2. Large Hadron Collider injector complex, with the SPS as preinjector, and the CERN proton synchrotron complex to prepare the beams for injection into the SPS.

(SPS), as shown in Fig. 2. In the SPS the beam is accelerated to the LHC injection energy of $450 \mathrm{GeV}$ and then transferred via about $3 \mathrm{~km}$ long transfer lines. One beam pulse from the SPS includes up to 288 bunches, therefore filling LHC requires about 10 SPS cycles for each beam.

Beam commissioning started in 2008. After a few weeks of operation, an interconnection between two superconducting magnets broke during magnet tests and a lengthy repair and consolidation campaign was necessary. Beam operation could continue late in 2009, with the energy limited to $3.5 \mathrm{TeV}$.

During 2011, the LHC operated with protons accelerated to $3.5 \mathrm{TeV}$. Each beam had up to 1380 bunches, with an intensity per bunch of up to $1.45 \times 10^{11}$. This corresponds to an energy stored in each beam of more than $110 \mathrm{MJ}$.

For each of the two LHC beams there is a beam dumping system that extracts the beam after nominal operation as well as in the case of equipment failure. The extracted beam is transferred through a $700 \mathrm{~m}$ long beam line to a graphite beam dump block. The extraction is fast and all bunches are extracted within one turn. Along the beam dump line the beam size increases to several $\mathrm{mm}$. The particle density is further reduced using two pulsed magnets, one horizontal and one vertical, that deflect each bunch differently to reduce the energy density to an acceptable level. When the beam reaches the dump block, the entire beam size is of the order of several $10 \mathrm{~cm}$. The beam dumping system is one of the important safety systems required during the LHC operation. Since the start of beam operation in 2009 , the machine protection systems, interlocks, and the beam dumping system performed as expected.

\section{THE HIRADMAT FACILITY AND THE SUPER PROTON SYNCHROTRON}

The Super Proton Synchrotron (SPS) is used as LHC injector, but also to accelerate and extract protons and ions (such as lead and other ion species) for fixed target experiments and for producing neutrinos (CNGS). In particular, the risks during the fast extraction of LHC and CNGS beams must be considered since any failure during this process can lead to serious equipment damage.

The SPS accelerator is $6.9 \mathrm{~km}$ long (circumference) and accelerates protons from $14 \mathrm{GeV} / c$ or $26 \mathrm{GeV} / c$ to a momentum of up to $450 \mathrm{GeV} / c$. It is a cycling machine with cycles having a length of about $15 \mathrm{~s}$. The transverse beam size is largest at injection and decreases with the square root of the beam energy during acceleration. For the operation as a synchrotron, the beam size is typically of the order of $1 \mathrm{~mm}$.

When the SPS operates as LHC injector, up to 288 bunches are accelerated, each bunch with about $1.15 \times 10^{11}$ protons (nominal parameters). The bunch length is $0.5 \mathrm{~ns}$ and two neighboring bunches are separated by $25 \mathrm{~ns}$ so that the duration of the entire beam is $7.2 \mu \mathrm{s}$. The normalized emittance is $3.75 \times 10^{-6} \mathrm{~m}$. Assuming a beta function of $100 \mathrm{~m}$, the beam size is $0.88 \mathrm{~mm}$. When the SPS was used as proton-antiproton collider, the luminosity was maximized by minimizing the beta function to $0.5 \mathrm{~m}$. Assuming this value, the beam size would be as small as $0.06 \mathrm{~mm}$.

The HiRadMat facility is dedicated to beam shock impact experiments. It is designed to allow testing of accelerator components, in particular those for LHC, to the

TABLE I. HiRadMat beam properties.

\begin{tabular}{lccc}
\hline \hline Parameter & Symbol & Protons & Pb ions \\
\hline Particle energy & $E$ & $440 \mathrm{GeV}$ & $36.9 \mathrm{TeV}$ \\
Bunch intensity & $N_{b}$ & $1.7 \times 10^{11}$ & $7 \times 10^{7}$ \\
Maximum number of bunches per pulse & $n_{\max }$ & 288 & 52 \\
Maximum pulse intensity & $N_{p}=n_{\max } \cdot N_{b}$ & $4.9 \times 10^{13}$ protons & $3.64 \times 10^{9}$ ions \\
Bunch spacing & $t_{b}$ & $25 \mathrm{~ns}$ & $100 \mathrm{~ns}$ \\
Minimum beam size (rms) & $\sigma_{\text {beam }}$ & $0.1 \mathrm{~mm}$ & $0.1 \mathrm{~mm}$ \\
rms bunch length & $\sigma_{z}$ & $11.24 \mathrm{~cm}$ & $11.24 \mathrm{~cm}$ \\
Pulse length & $t_{p}$ & $7.2 \mu \mathrm{s}$ & $5.2 \mu \mathrm{s}$ \\
\hline \hline
\end{tabular}


TABLE II. Overview of SPS calculations.

\begin{tabular}{|c|c|c|c|}
\hline \multicolumn{3}{|c|}{$\mathrm{Cu}$ target } & $\mathrm{W}$ target \\
\hline $\begin{array}{l}\text { FLUKA: Energy deposition } \\
\text { at solid density. BIG2: } \\
\text { Hydrodynamic calcula- } \\
\text { tions along radius at one } \\
\text { fixed point on axis [26] }\end{array}$ & $\begin{array}{l}\text { Hydrodynamic calcula- } \\
\text { tions in r-Z geometry, } \\
\text { energy deposition in BIG2 } \\
\text { normalized with axial line } \\
\text { density (analytic approxi- } \\
\text { mation) [27] }\end{array}$ & $\begin{array}{l}\text { Hydrodynamic calcula- } \\
\text { tions in r-Z geometry } \\
\text { running FLUKA and BIG2 } \\
\text { codes iteratively (this pa- } \\
\text { per, Sec. VI). }\end{array}$ & $\begin{array}{cc}\text { FLUKA: Energy depo- } & \text { Hydrodynamic calcula- } \\
\text { sition at solid density. } & \text { tions in r-Z geometry, } \\
\text { BIG2: Hydrodynamic } & \text { energy deposition in BIG2 } \\
\text { calculations along ra- } & \text { normalized with axial line } \\
\text { dius at one fixed point } & \text { density (analytic approxi- } \\
\text { on axis [26] } & \text { mation) [19] }\end{array}$ \\
\hline
\end{tabular}

TABLE III. Overview of LHC calculations.

\begin{tabular}{|c|c|c|c|}
\hline \multicolumn{2}{|l|}{$\mathrm{Cu}$ target } & \multicolumn{2}{|c|}{$\mathrm{C}$ target } \\
\hline $\begin{array}{l}\text { FLUKA: Energy deposition at } \\
\text { solid density. BIG2: } \\
\text { Hydrodynamic calculations } \\
\text { along radius at one fixed } \\
\text { point on axis [1] }\end{array}$ & $\begin{array}{l}\text { Hydrodynamic calculations } \\
\text { in } \mathrm{r}-\mathrm{Z} \text { geometry, energy dep- } \\
\text { osition in BIG2 normalized } \\
\text { with axial line density (ana- } \\
\text { lytic approximation) [6] }\end{array}$ & $\begin{array}{l}\text { Hydrodynamic calculations } \\
\text { in } \mathrm{r}-\mathrm{Z} \text { geometry, energy dep- } \\
\text { osition in BIG2 normalized } \\
\text { with axial line density (ana- } \\
\text { lytic approximation) [28] }\end{array}$ & $\begin{array}{l}\text { Hydrodynamic calculations } \\
\text { in } r-Z \text { geometry running } \\
\text { FLUKA and BIG2 codes itera- } \\
\text { tively [29] and (this paper, } \\
\text { Sec. V) }\end{array}$ \\
\hline
\end{tabular}

impact of high intensity pulsed beams. It has been constructed and commissioned during 2010-2011 [11-13]. Beam properties are shown in Table I. It will provide a $440 \mathrm{GeV}$ proton beam or a $36.9 \mathrm{TeV}$ lead ion beam. For protons, the beam can allocate up to 288 bunches, each comprising of up to $1.7 \times 10^{11}$ particles. The focal size can go down to $0.1 \mathrm{~mm} \sigma_{\text {rms }}$, thus providing a very dense beam (energy/size). The size can be tuned from 0.1 to $2 \mathrm{~mm}$. The experimental area has a length of more than $9 \mathrm{~m}$ that allows up to three experiments to be installed in parallel.

The primary purpose of the experiments suggested in this paper is to validate the existence of hydrodynamic tunneling that results from the hydrodynamic effects and to gain confidence with the methodology and simulation tools used. At later stages, other areas of research, for example, warm dense matter (WDM) and HED physics [14-25], may also benefit from this unique facility.

\section{OVERVIEW OF SPS AND LHC CALCULATIONS}

During the past few years, a large number of simulations were performed for SPS and LHC beams, using different methodologies and for different target materials. Before presenting the latest simulation method and results for LHC carbon targets and SPS copper targets, Tables II and III summarize all different combinations of methodologies and materials used in the past.

\section{SIMULATIONS OF THE LHC BEAM WITH SOLID CARBON CYLINDER}

In this section we present the numerical simulations of the thermodynamic and hydrodynamic behavior of a solid carbon cylindrical target that is impacted by the full LHC beam. These calculations have been done in two steps.
First, the energy deposition in the target from protons is calculated using the FLUKA code [3,4], assuming solid carbon density. FLUKA is an established particle interaction and Monte Carlo package capable of simulating all components of the particle cascades in matter, up to multi-TeV energies. Second, this energy deposition data is used as input to a sophisticated two-dimensional hydrodynamic code, BIG2 [2], to calculate the beam-target interaction that causes hydrodynamic motion which leads to density reduction at the target center.

Multiphase, multicomponent equation of state (EOS) data from [30] is used in BIG2 to model different physical states of graphite in the calculations. The modified density distribution obtained from the BIG2 code is used back in the FLUKA code to calculate the corresponding modified energy deposition distribution for bunches arriving later. This new distribution is then used in the BIG2 code as the next step. In this manner, the two codes are run iteratively. The iteration interval is determined by the time during which the target density is reduced by $15 \%-20 \%$ due to hydrodynamic effects. The typical iteration step used in these simulations was $2.5 \mu \mathrm{s}$. The results are presented below.

An accidental loss of even a small fraction of the LHC beam energy can severely damage the equipment. One may therefore imagine some of the hazards, e.g., if the entire beam is lost at a single point. The likelihood of occurrence of such an accident is quite remote, nevertheless it is important to quantify the consequences. One of the failure modes is a wrong deflection of the beam by the extraction kicker. A carbon absorber with a length of $6 \mathrm{~m}$, the TCDQ, is installed to capture the particles. It is planned to install an even longer absorber in the future. One objective of the simulations presented in this section is to address the question if such an absorber can absorb the entire beam if the extraction kicker deflects the beam by a wrong angle. 


\section{A. Proton energy deposition calculations using FLUKA}

For the study presented in this paper, the geometry for the FLUKA calculations is a cylinder of solid carbon with radius $=5 \mathrm{~cm}$ and length $=6 \mathrm{~m}$. The solid density of carbon is assumed to be $2.28 \mathrm{~g} / \mathrm{cm}^{3}$. The energy deposition is obtained using a two-dimensional Gaussian beam distribution (horizontal and vertical $=$ with $\sigma_{\text {rms }}=$ $0.5 \mathrm{~mm}$ ) that was incident perpendicular to the front face of the cylinder. This beam size was selected for the simulation since it corresponds to the size of the beam at beam absorbers downstream of the extraction kicker, which is the most likely point of impact in case of a failure.

In Fig. 3(a) we present the energy deposition distribution per $7 \mathrm{TeV}$ proton in units $\mathrm{GeV} / \mathrm{g}$ as calculated by FLUKA assuming solid material density. This data shows that the range of the shower is about $3 \mathrm{~m}$ in the target and the peak
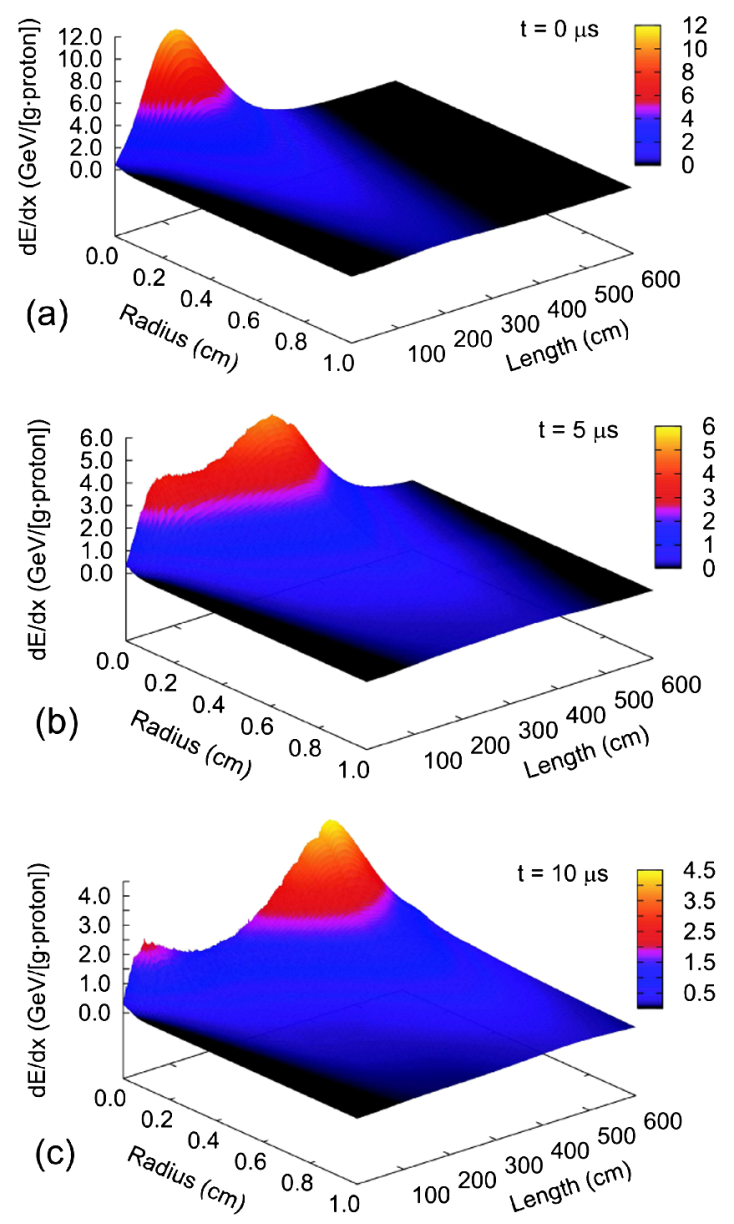

FIG. 3. FLUKA calculations of energy deposition of a single $7 \mathrm{TeV}$ LHC proton in a solid carbon cylinder having radius $r=$ $5 \mathrm{~cm}$, length $L=6 \mathrm{~m}$, with facial irradiation, beam spot size characterized by standard deviation $\sigma=0.5 \mathrm{~mm}$; (a) using solid density of $2.28 \mathrm{~g} / \mathrm{cm}^{3}$; (b) using the density distribution provided by the BIG2 at $t=5 \mu \mathrm{s}$ (second iteration); and (c) using the density distribution provided by the BIG2 at $t=10 \mu \mathrm{s}$ (fourth iteration). of the distribution is around $12 \mathrm{GeV} / \mathrm{p} / \mathrm{g}$. The FLUKA calculations also suggest that approximately $54 \%$ beam energy escapes while $46 \%$ is absorbed in the target.

Figure 3(b) presents the energy deposition data obtained with FLUKA, but using the density distribution provided by BIG2 at $t=5 \mu \mathrm{s}$ (second iteration). The energy deposition distribution has been substantially modified with a significant broadening of the energy peak that indicates deeper penetration of the protons and the shower into the target. Moreover, the distribution has two peaks and the higher peak lies in the beam direction where the material density is much higher.

The energy deposition distribution plotted in Fig. 3(c) has been calculated by FLUKA using the density distribution obtained from BIG2 at $t=10 \mu \mathrm{s}$ (fourth iteration). This figure shows a much longer penetration of the particle shower and the contrast between the two peaks is much more pronounced.

\section{B. Hydrodynamic simulations using BIG2}

In the first iteration of the BIG2 simulations we consider the same solid carbon cylindrical target. The FLUKA energy deposition data presented in Fig. 3(a) is used as input to BIG2 and the calculations are carried out until the density in the beam heated region changes by about $15 \%-20 \%$.

This condition leads to an iteration step of $2.5 \mu \mathrm{s}$. In Fig. 4(a), the specific energy deposition distribution is plotted in the $\mathrm{r}-\mathrm{Z}$ plane of the target at $t=5 \mu \mathrm{s}$ (second iteration), when 200 proton bunches have been delivered. It is seen that the peak specific energy deposition is about $30 \mathrm{~kJ} / \mathrm{g}$ and the beam heated region has extended to about $4 \mathrm{~m}$, compared to around $3 \mathrm{~m}$ in the solid target. This extension in the length of the energy deposition zone is due to the hydrodynamic tunneling of the protons and the shower particles due to the density drop along the target axis caused by hydrodynamic effects.

Figures 4(b) and 4(c) present the same variable as 4(a), but at $t=10 \mu \mathrm{s}$ and $t=15 \mu \mathrm{s}$, respectively. It is seen in Fig. 4(b) that the length of the heated zone has been extended to about $5 \mathrm{~m}$ and the peak specific energy is $40 \mathrm{~kJ} / \mathrm{g}$. By this time, 400 proton bunches have been delivered, but the increase in the maximum value of the specific energy is slow compared to that in Fig. 4(a). This is due to the fact that the volume over which the beam energy is distributed is continuously increasing with time and therefore the maximum specific energy deposition does not increase linearly with the number of bunches. This effect is more pronounced in Fig. 4(c) where after the delivery of 600 proton bunches the maximum specific energy increases only to $45 \mathrm{~kJ} / \mathrm{g}$ whereas the beam penetrates the entire length of the target. We also note that the energy deposition is a local phenomenon (heat conduction is weak at such high densities on the time scale of interest), we therefore only show the inner $2 \mathrm{~cm}$ radius of the target in Fig. 4. 


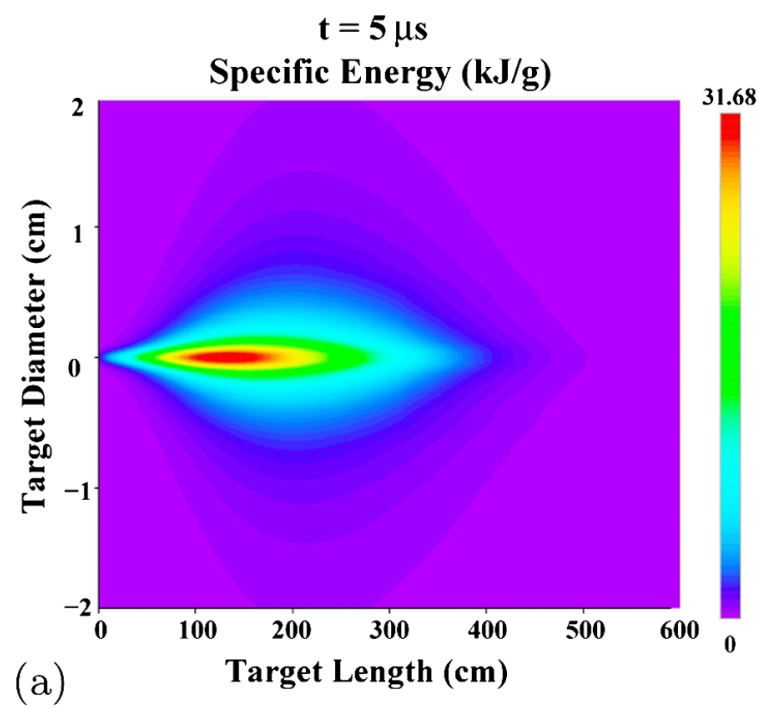

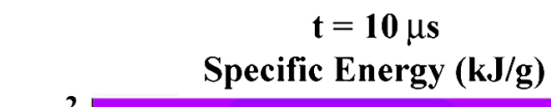
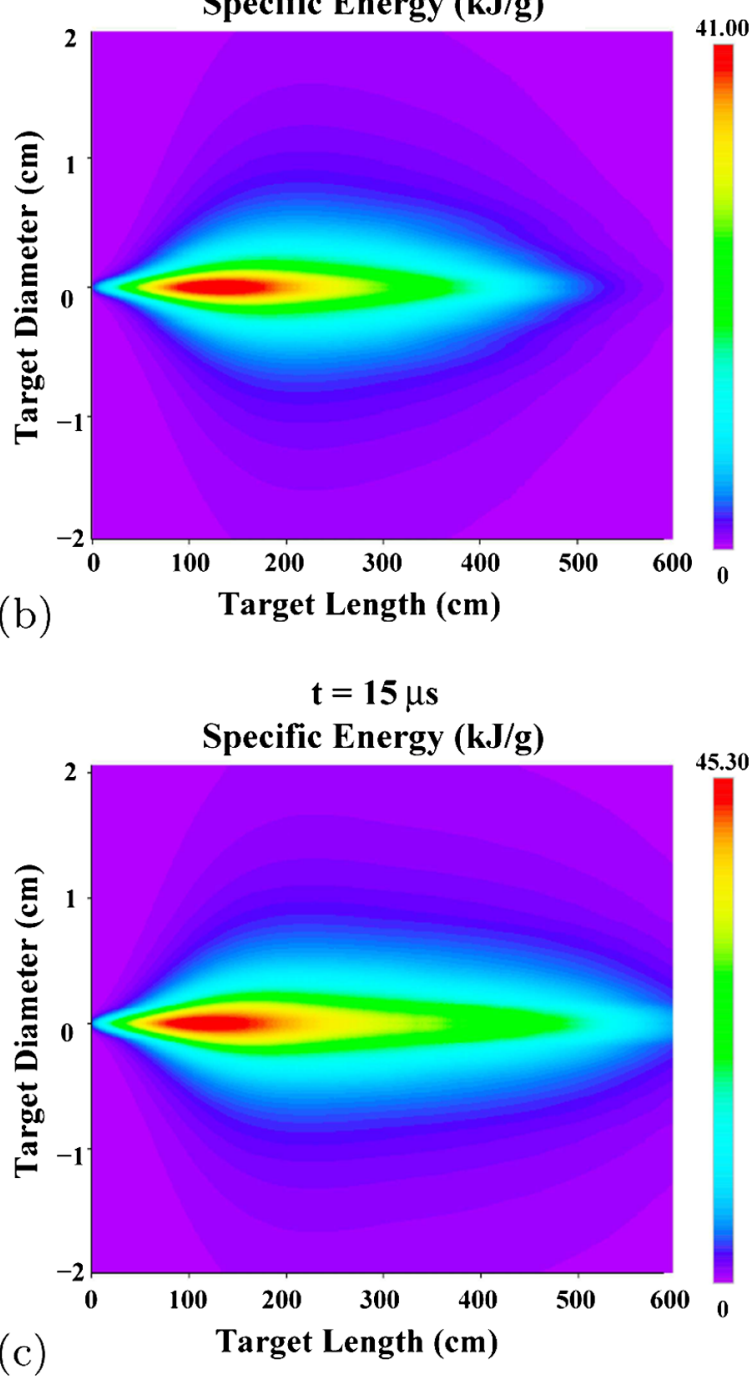

FIG. 4. Specific energy deposition distribution calculated by BIG2 in the carbon cylinder, $r=5 \mathrm{~cm}, L=6 \mathrm{~m}$, irradiated by the LHC beam at the left face; (a) at $t=5 \mu \mathrm{s}$; (b) at $t=10 \mu \mathrm{s}$; and (c) at $t=15 \mu \mathrm{s}$.

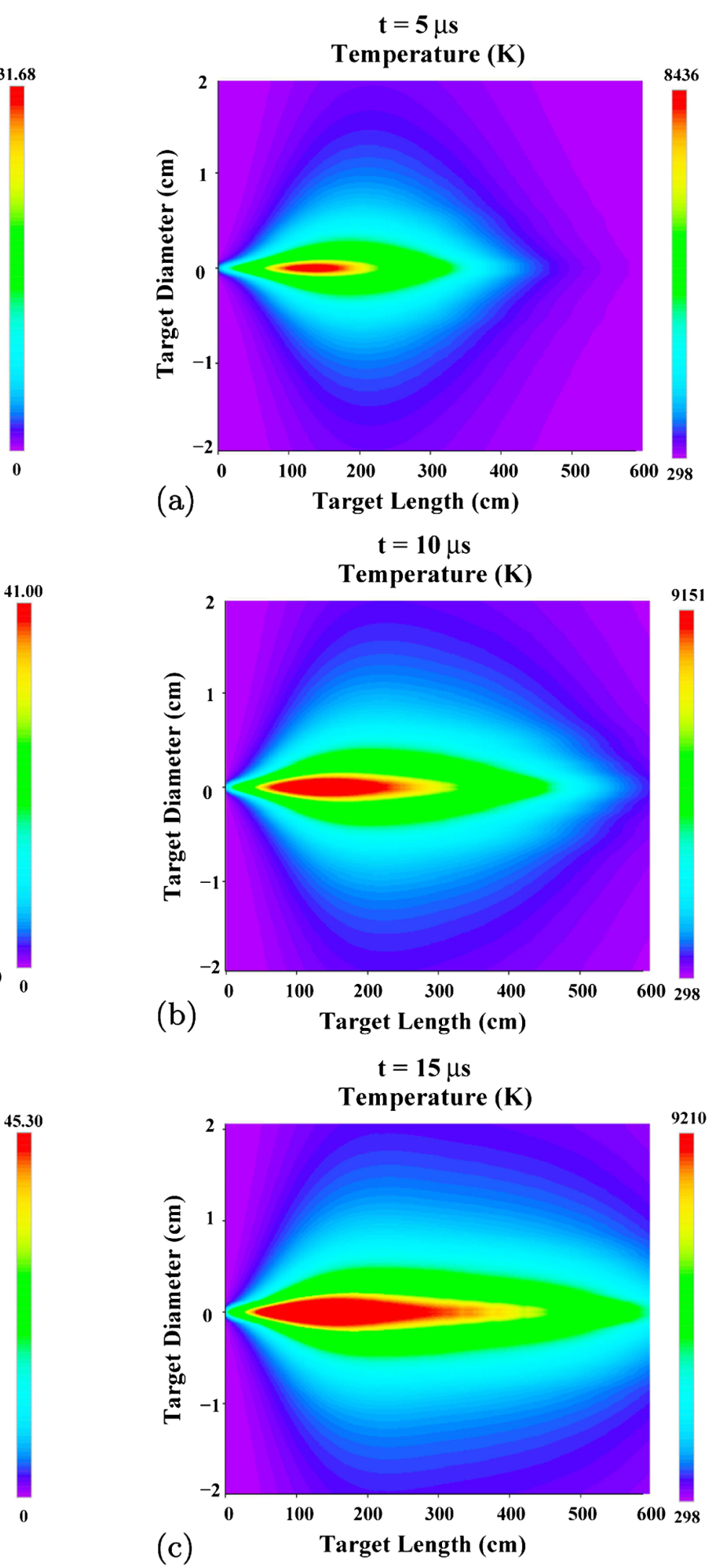

FIG. 5. Temperature distribution calculated by BIG2 in the carbon cylinder, $r=5 \mathrm{~cm}, L=6 \mathrm{~m}$, irradiated by the LHC beam at the left face; (a) at $t=5 \mu \mathrm{s}$; (b) at $t=10 \mu \mathrm{s}$, and (c) at $t=15 \mu \mathrm{s}$. 

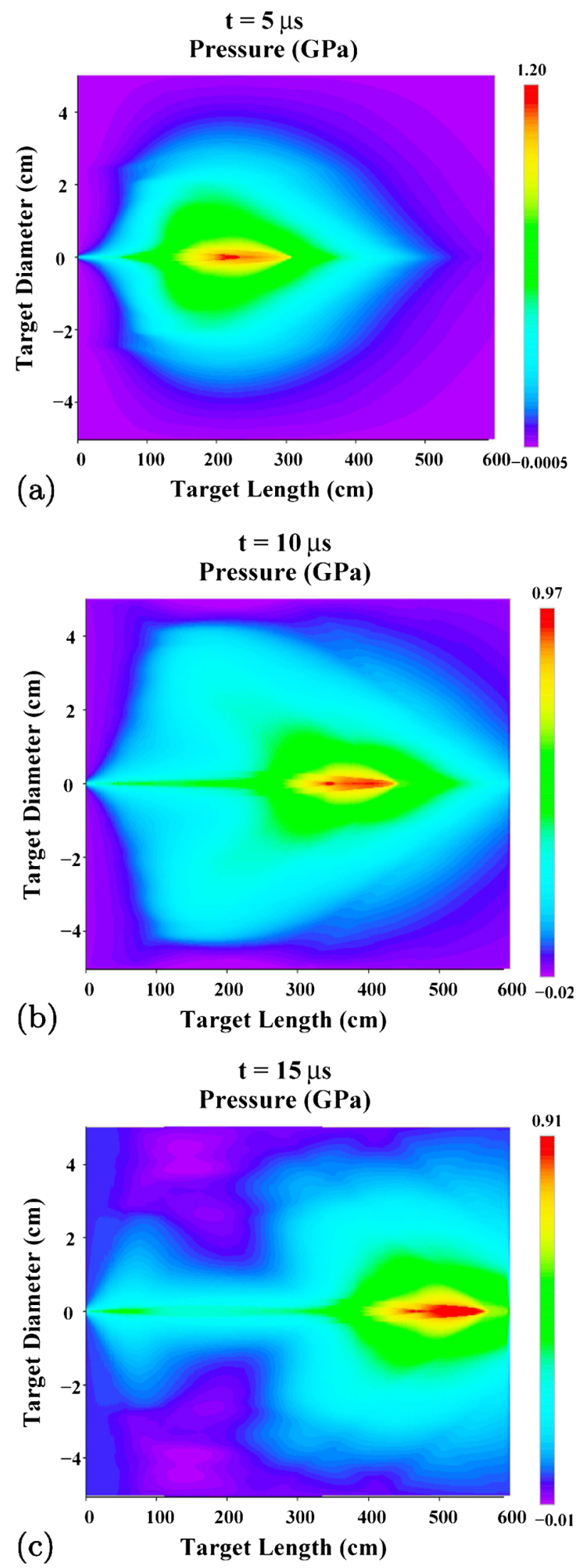

FIG. 6. Pressure distribution calculated by BIG2 in the carbon cylinder, $r=5 \mathrm{~cm}, L=6 \mathrm{~m}$, irradiated by the LHC beam at the left face; (a) at $t=5 \mu \mathrm{s}$; (b) at $t=10 \mu \mathrm{s}$; and (c) at $t=15 \mu \mathrm{s}$. $\mathbf{t}=\mathbf{5} \mu \mathrm{s}$

Density $\left(\mathrm{g} / \mathrm{cm}^{3}\right)$

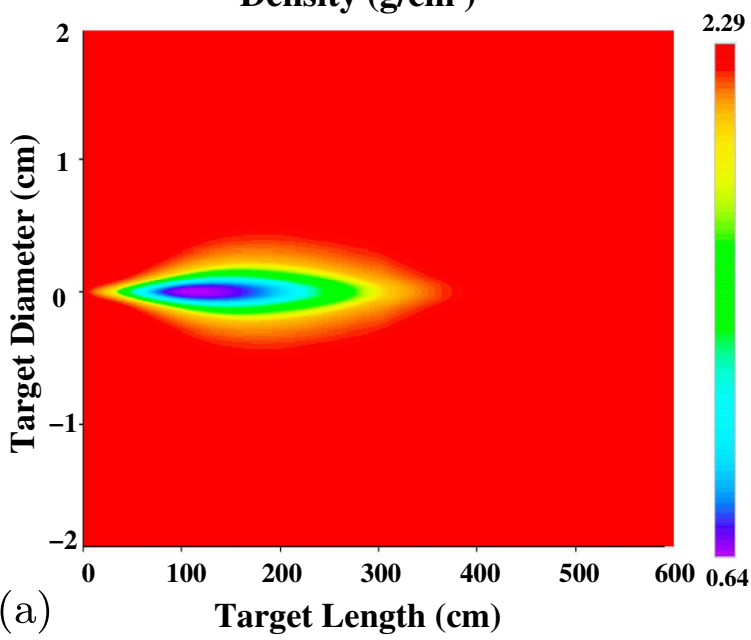

$\mathbf{t}=\mathbf{1 0} \mu \mathrm{s}$

Density $\left(\mathrm{g} / \mathrm{cm}^{3}\right)$

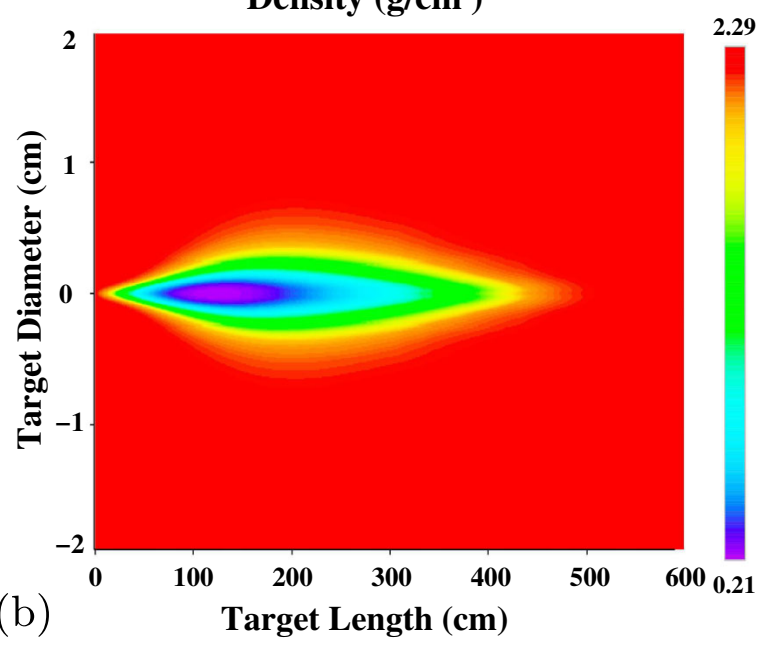

$\mathbf{t}=15 \mu \mathrm{s}$

Density $\left(\mathrm{g} / \mathrm{cm}^{3}\right)$

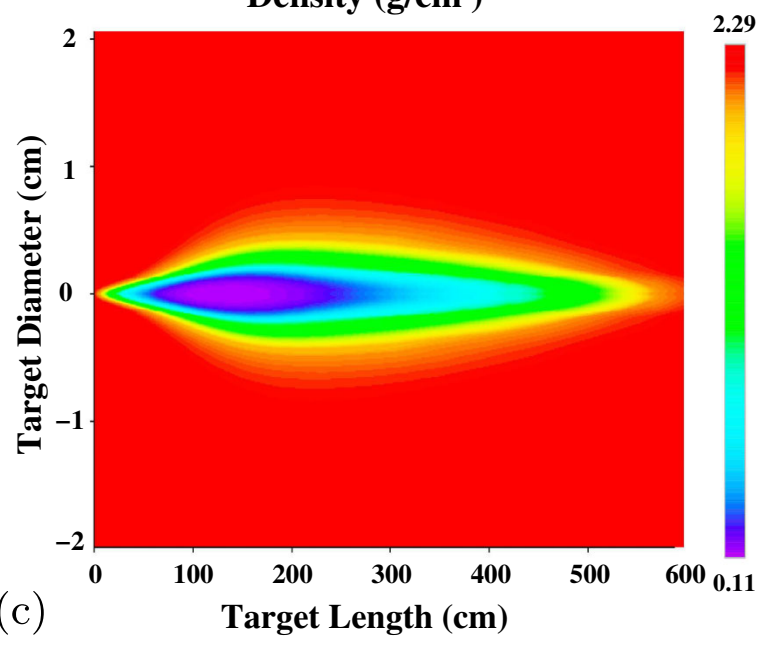

FIG. 7. Density distribution calculated by BIG2 in the carbon cylinder, $r=5 \mathrm{~cm}, L=6 \mathrm{~m}$, irradiated by the LHC beam at the left face; (a) at $t=5 \mu \mathrm{s}$; (b) at $t=10 \mu \mathrm{s}$; and (c) at $t=15 \mu \mathrm{s}$. 
The target material is heated due to the energy deposition by the beam that leads to substantial rise in the temperature in the deposition volume. In Figs. 5(a)-5(c) we present the temperature distribution in the target calculated by BIG2 corresponding to the energy deposition distributions in Figs. 4(a)-4(c), respectively.

It is seen in Fig. 5(a) that the maximum temperature along the target axis at $t=5 \mu \mathrm{s}$ is of the order of $8400 \mathrm{~K}$, which means that the material in this region is in a gaseous state. Further it is seen in Fig. 5(b) that, at $t=10 \mu \mathrm{s}$, the maximum temperature slightly increases to around $9100 \mathrm{~K}$ and the length of the heated region increases according to Fig. 4(b). Finally, Fig. 5(c) shows that further temperature increase at $t=15 \mu$ is nominal and in fact the maximum temperature saturates around $9000 \mathrm{~K}$. It is also seen that by this time the heating extends through the entire length of the target. This shows that the material in this region is severely damaged and is in the WDM regime [14-25].

The high temperature in the beam heated zone generates very high pressure that drives outmoving radial compression waves which transport material outwards, thereby causing density depletion along and around the target axis. In Figs. 6(a)-6(c) we plot the pressure distribution in the target calculated by BIG2 corresponding to the temperature distribution presented in Figs. 5(a)-5(c), respectively.

It is seen in Fig. 6(a) that a maximum pressure of 1.2 GPa exists at the target axis at $t=5 \mu \mathrm{s}$ and formation of a radially outgoing compression wave is also seen. Since the pressure generates compression waves that propagate throughout the target (nonlocal phenomenon), we show the pressure in the full radius of $5 \mathrm{~cm}$ of the target. Figure 6(b) shows that at $t=10 \mu \mathrm{s}$ the maximum value of pressure has been reduced to $0.97 \mathrm{GPa}$, but the pressure peak has significantly moved along the target axis. This again is because the beam penetrates deeper along the axis. It is also seen that around $L=2 \mathrm{~m}$, the radial compression wave has already been reflected at the target surface and a slight negative pressure is generated near the boundary which represents a restoring force against the outward radial motion of the cylinder surface. Figure 6(c) shows that the pressure peak of $0.9 \mathrm{GPa}$ has arrived at the opposite face of the cylinder due to the penetration of the beam through the entire cylinder length of $6 \mathrm{~m}$.

The density distributions corresponding to Figs. 6(a)-6 (c) are presented in Figs. 7(a)-7(c), respectively. It is seen in Fig. 7(a) (at $t=5 \mu \mathrm{s}$ ) that, due to the radial outgoing compression wave generated by the pressure distribution shown in Fig. 6(a), the density at the inner part of the target along the axis has already been reduced to $0.64 \mathrm{~g} / \mathrm{cm}^{3}$. Figure 7(b) shows a further density reduction in this region with a minimum density of $0.21 \mathrm{~g} / \mathrm{cm}^{3}$. The size of the depletion region increases in the longitudinal direction as well. It is further seen in Fig. 7(c) that, at $t=15 \mu \mathrm{s}$, the minimum density has become $0.11 \mathrm{~g} / \mathrm{cm}^{3}$ and the beam has penetrated through the entire length of the cylinder.
To have a more quantitative assessment of the parameters, we plot the specific energy and the temperature along the target axis, at different times, in Figs. 8(a) and 8(b), respectively. It can be seen in Fig. 8(a) that a high value of the specific energy with a peak value of about $25 \mathrm{~kJ} / \mathrm{g}$ is achieved at $t=4 \mu \mathrm{s}$. However, the rate of increase in the value of this parameter slows down with time and at $t=$ $12 \mu \mathrm{s}$ the value saturates around $40 \mathrm{~kJ} / \mathrm{g}$. This is due to the fact that the deposition volume continuously increases with time as the hydrodynamic tunneling of the protons and the shower goes on.

The temperature curve shows a very interesting behavior. The curve labeled with $4 \mu \mathrm{s}$ has a hump shaped part between $L=100$ and $200 \mathrm{~cm}$ which represents the gaseous phase of carbon. The constant temperature regions extending towards the left and the right of the hump, on the other hand, represent the two-phase liquid-gas state of the material. The two-phase region on the right side of the hump continuously moves towards the right due to the higher energy deposition as a result of deeper penetration
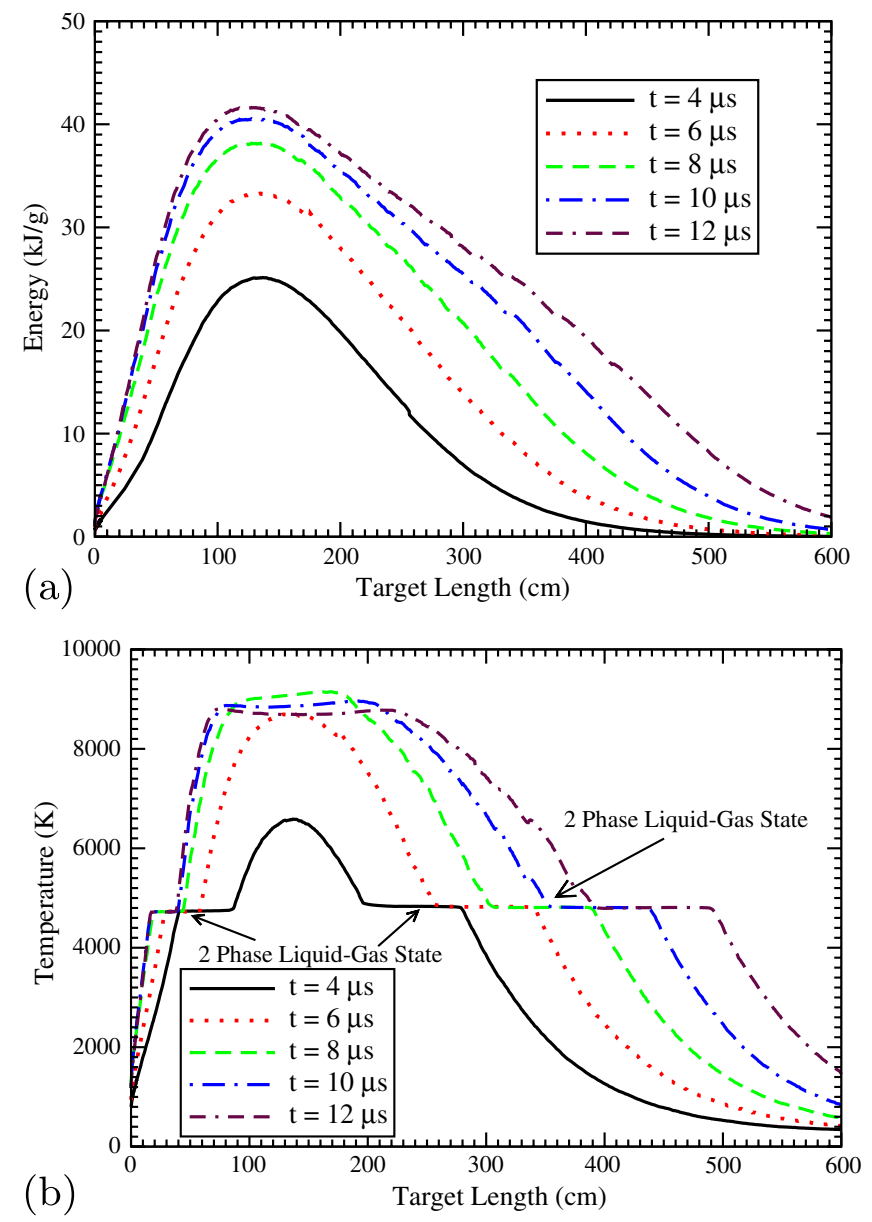

FIG. 8. (a) Specific energy deposition along the target axis at different times. (b) Temperature along the target axis at different times. 
of the beam. The temperature in the gaseous region increases with time and saturates around $9000 \mathrm{~K}$.

The pressure, the density, and the surface velocity along the target axis are plotted for different times in Figs. 9(a)-9(c), respectively.

Figure 9(a) shows that the peak in the pressure profile continuously moves towards the right as a result of the hydrodynamic tunneling effect.
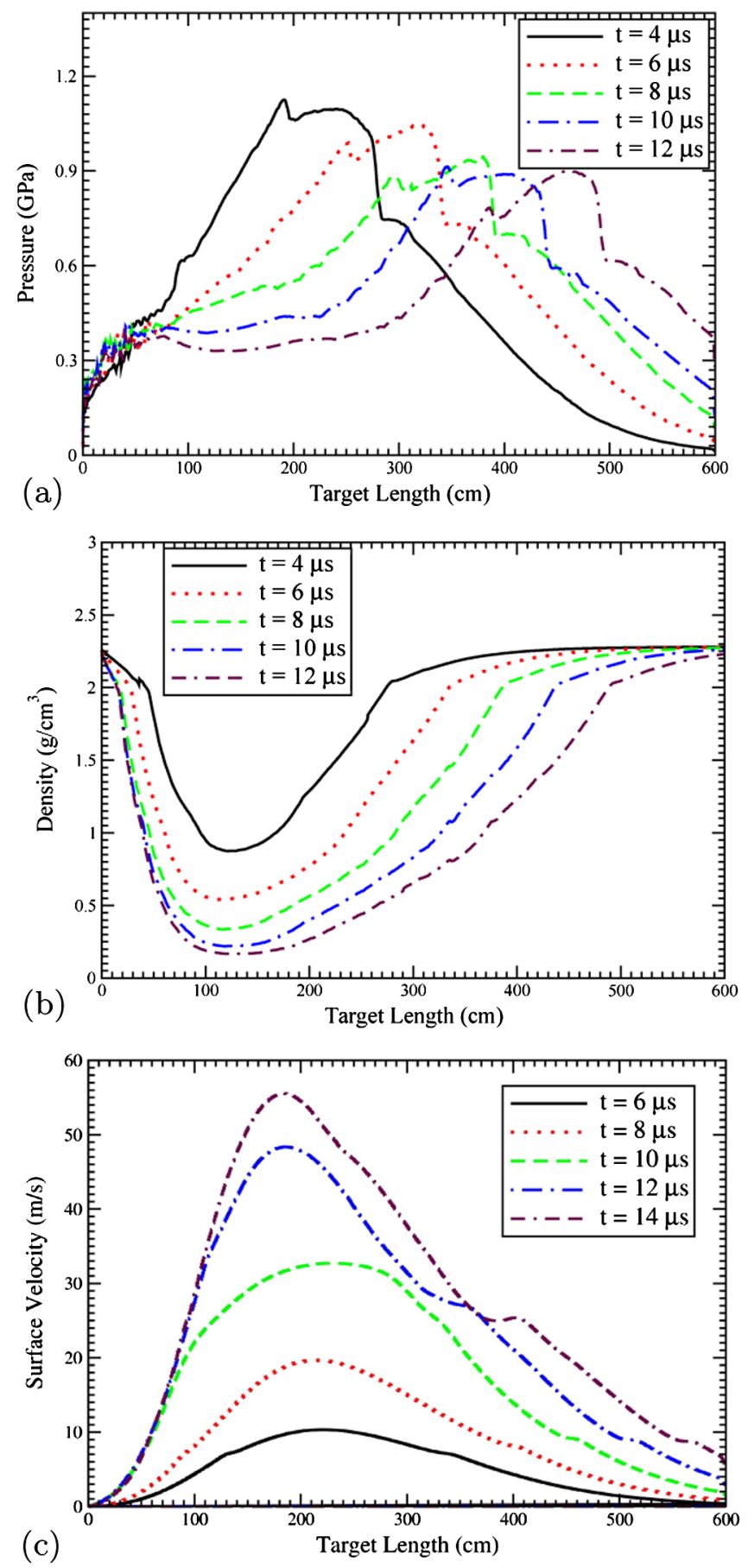

FIG. 9. (a) Pressure along the target axis at different times; (b) density along the target axis at different times; and (c) surface velocity along the target length at different times.
The density profiles plotted in Fig. 9(b) show that after $6 \mu \mathrm{s}$, the density depletion front moves along the axis with a constant speed of about $25 \mathrm{~cm} / \mu \mathrm{s}$. This means that during the remaining $83 \mu \mathrm{s}$ when the other bunches hit the target, the penetration depth will be about $21 \mathrm{~m}$. Since in the first $6 \mu \mathrm{s}$, the beam has penetrated up to $4 \mathrm{~m}$, the total penetration depth will be around $25 \mathrm{~m}$. This suggests that the $6 \mathrm{~m}$ length of the TCDQ beam stopper is not sufficient to stop the beam completely, nevertheless, the beam will be significantly weakened after emerging through this stopper and therefore the damage to other equipment will be reduced.

When the compression wave that propagates radially outwards arrives at the cylinder surface, it imparts a velocity onto the surface. In Fig. 9(c), we present the profiles of the surface velocity at different times. It is seen that the profile shape is in accordance with the energy deposition distribution, as a peak in the surface velocity exists at the same location where the peak in the energy deposition is located in the target.

The maximum value of the surface velocity at $t=6 \mu \mathrm{s}$ is about $10 \mathrm{~m} / \mathrm{s}$ that increases to around $20 \mathrm{~m} / \mathrm{s}$ at $t=$ $8 \mu \mathrm{s}$. Later, at $t=10 \mu \mathrm{s}$ the maximum value of the surface velocity increases to about $30 \mathrm{~m} / \mathrm{s}$ and the peak becomes much broader because the pressure wave broadens due to the deeper penetration of the projectile particles into the target. At $t=12 \mu \mathrm{s}$, the maximum surface velocity increases to about $50 \mathrm{~m} / \mathrm{s}$, but the increase in the next $2 \mu \mathrm{s}$ interval is much slower, as it is seen that at $t=14 \mu \mathrm{s}$ the maximum value of the surface velocity is around $55 \mathrm{~m} / \mathrm{s}$.

This is because the tensile force generated by the yield strength of the material finally decelerates the outmoving surface and tends to restore it to its original position. If the material is in an elastic state, the target boundary will oscillate around an equilibrium position. In order to check this effect, we evaluated the von Mises parameter, $M$, in this region. It is to be noted that in a two-dimensional approximation,

$$
M=\left[3\left(\sigma_{x x}^{2}+2 \sigma_{x y}^{2}+\sigma_{y y}^{2}\right) /\left(2 Y_{0}^{2}\right)\right],
$$

where $\sigma$ 's represent different components of the stress tensor and $Y_{0}$ is the yield strength of graphite which is $70 \mathrm{MPa}$ in our case. These calculations show that in the solid part of the target, $M$ is safely below 1 and the material therefore remains in an elastic state. If the calculations are continued for a much longer time, one will see that the surface velocity will change its sign and the surface will start moving inwards.

Similar calculations were also done for the interaction of the Superconducting Super Collider $20 \mathrm{TeV}$ proton beam with a solid carbon beam dump [31]. In that case the transverse particle intensity distribution in the focal spot was characterized with $\sigma=2.0 \mathrm{~mm}$, which is 4 times larger than that used in our calculations. Two different 
particle fluences, namely $4.5 \times 10^{17}$ and $1.0 \times$ $10^{19}$ particles/s, were considered. Since the pulse duration was $290 \mu \mathrm{s}$, the total number of protons in the beam corresponding to the above two fluences were $1.3 \times 10^{14}$ and $2.9 \times 10^{15}$, respectively. The calculations reported in [31] have shown that the beam will penetrate at a speed of $7 \mathrm{~cm} / \mu \mathrm{s}$ in the case of the lower fluence and at $70 \mathrm{~cm} / \mu \mathrm{s}$ in the case of the higher fluence. These results are very similar to our calculations.

\section{SIMULATIONS OF THE SPS BEAM WITH SOLID COPPER CYLINDER}

Previously, we have done extensive simulations of the heating and hydrodynamic response of solid targets of different materials that were irradiated with the SPS beam. In the first phase of these studies we considered the hydrodynamic effects in the radial direction at the point of maximum energy deposition on the target axis. Tungsten and copper targets were considered. In both cases the energy deposition calculated for solid density by FLUKA was used [28]. In the second stage, we studied the heating and the hydrodynamic behavior of a solid tungsten cylinder in the $\mathrm{r}-\mathrm{Z}$ geometry, but still used the solid-density energy deposition data [32]. The hydrodynamic tunneling effect could not be simulated in these calculations. This work was further improved by normalizing the solid-density energy deposition data by the line density along the target axis at every time step and in every simulation cell of the BIG2 code $[19,27]$. This analytic approximation showed significant lengthening of the proton beam range in the target and thus helped to understand this problem in the case of the SPS beam. The penetration length of the projectiles and the shower substantially increased when the beam spot was reduced because of the higher specific power deposition.

Recently, we have made a significant improvement by simulating the above problem using the FLUKA and the BIG2 codes, iteratively. Three different focal spot sizes with $\sigma=$ $0.1,0.2$, and $0.5 \mathrm{~mm}$, respectively, have been considered while the target is a solid copper cylinder. The experiment planned at HiRadMat will be performed with these parameters. In this paper we only present the calculations of the case of $\sigma=0.5 \mathrm{~mm}$. An iteration time of $1 \mu \mathrm{s}$ is used in these simulations.

\section{A. Proton energy deposition calculations using FLUKA}

The geometry for the FLUKA calculations was a cylinder of solid copper with radius of $5 \mathrm{~cm}$ and length of $1.5 \mathrm{~m}$. The solid density of copper is $8.93 \mathrm{~g} / \mathrm{cm}^{3}$. The energy deposition is obtained using a realistic two-dimensional beam distribution, a Gaussian beam (horizontal and vertical $\sigma_{\mathrm{rms}}=0.5 \mathrm{~mm}$ ) that was incident perpendicular to the front face of the cylinder.
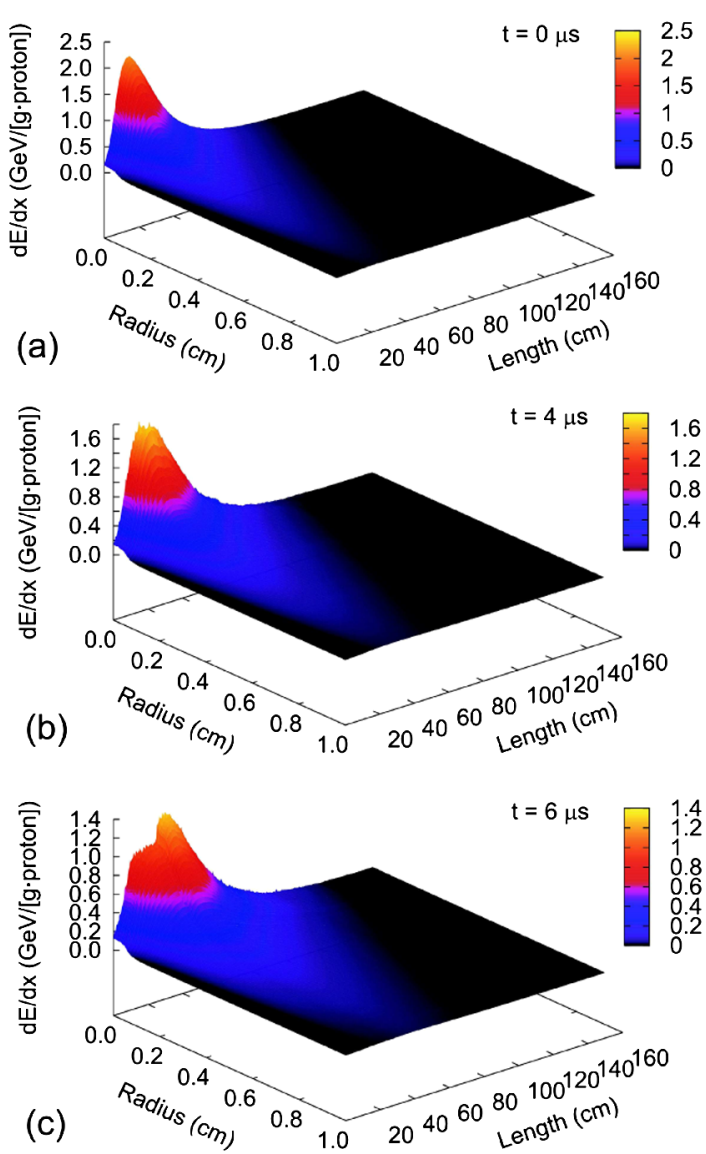

FIG. 10. FLUKA calculations of energy deposition of a single $440 \mathrm{GeV}$ SPS proton in a solid copper cylinder having radius $r=5 \mathrm{~cm}$, length $L=1.5 \mathrm{~m}$, with facial irradiation, beam spot size characterized by standard deviation $\sigma=0.5 \mathrm{~mm}$; (a) using solid density of $8.93 \mathrm{~g} / \mathrm{cm}^{3}$; (b) using the density distribution provided by the BIG2 at $t=4 \mu \mathrm{s}$ (fourth iteration); and (c) using the density distribution provided by the BIG2 at $t=6 \mu \mathrm{s}$ (sixth iteration).

In Fig. 10(a) we present the energy deposition per $440 \mathrm{GeV}$ proton in units $\mathrm{GeV} / \mathrm{g}$ as calculated by FLUKA assuming solid material density. This data shows that the range of the shower is about $75 \mathrm{~cm}$ in the target and the peak value of the distribution is around $2.1 \mathrm{GeV} / \mathrm{p} / \mathrm{g}$. The FLUKA calculations also suggest that approximately $36 \%$ beam energy escapes while $64 \%$ is absorbed in the target.

Figure 10(b) presents the energy deposition obtained with the FLUKA code, but using the density distribution provided by BIG2 at $t=4 \mu \mathrm{s}$ (fourth iteration). The energy deposition distribution has been modified with slight broadening of the energy peak.

The energy deposition plotted in Fig. 10(c) has been calculated by FLUKA using the density distribution obtained from BIG2 at $t=6 \mu \mathrm{s}$ (sixth iteration). This figure shows that the energy peak has moved deeper into the target and the value of the energy peak has been reduced to $1.4 \mathrm{GeV} / \mathrm{p} / \mathrm{g}$. These effects are much more pronounced in the cases of $\sigma=0.2$ and $0.1 \mathrm{~mm}$ beams. 


\section{B. Hydrodynamic simulations using BIG2}

The FLUKA energy deposition presented in Fig. 10(a) is used as input to BIG2. The calculations are carried out until the density in the beam heated region changes by about $15 \%-20 \%$. This consideration leads to an iteration step of $1 \mu \mathrm{s}$ and the calculations are done until all bunches impacted on the target at $t=7.2 \mu \mathrm{s}$. A semiempirical EOS data [33-35] has been used to treat different phases of copper in the simulations.

In Fig. 11(a) the specific energy deposition in the $\mathrm{r}-\mathrm{Z}$ plane of the target at $t=7.2 \mu \mathrm{s}$ is shown when all the 288 proton bunches have been delivered. The peak specific energy deposition is about $7 \mathrm{~kJ} / \mathrm{g}$, which is high enough to damage the target material. The profiles of the specific energy deposition along the axis at different times are plotted in Fig. 11(b). Between $t=1-3 \mu \mathrm{s}$, the maximum specific energy increases from about $1.5 \mathrm{~kJ} / \mathrm{g}$ to almost $4 \mathrm{~kJ} / \mathrm{g}$. In the interval $t=5-7.2 \mu \mathrm{s}$, on the other hand, the specific energy deposition increases from $6 \mathrm{~kJ} / \mathrm{g}$ to about $7 \mathrm{~kJ} / \mathrm{g}$. The reason for reduction in the rate of increase of the specific energy during the later part of the pulse is the deeper penetration of the beam into the target that results in distribution of the beam energy over an increasing volume.
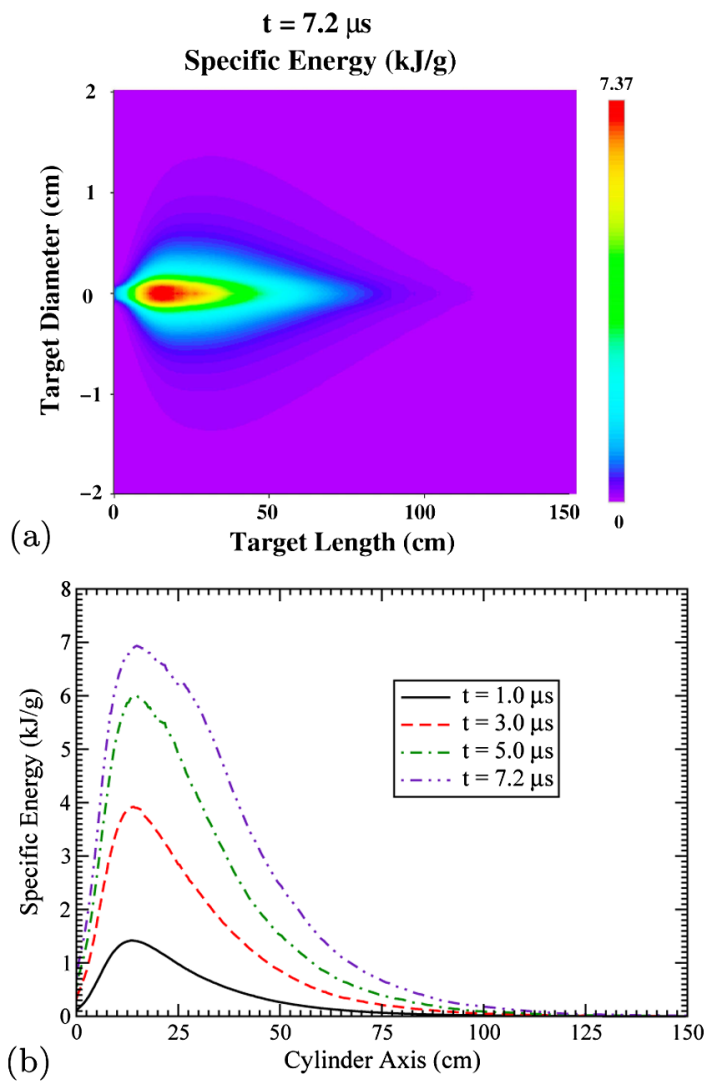

FIG. 11. (a) Specific energy distribution in the cylindrical copper target, $r=5 \mathrm{~cm}, L=1.5 \mathrm{~m}$, irradiated by the $440 \mathrm{GeV}$ SPS proton beam with $\sigma=0.5 \mathrm{~mm}$ at $t=7.2 \mu \mathrm{s}$ (end of the beam) and (b) specific energy deposition profiles along the axis at different times
Initially, the energy deposition region extends to about $75 \mathrm{~cm}$ along the axis and increases to about $100 \mathrm{~cm}$ at the end of the pulse.

The temperature distribution in the target corresponding to Fig. 11(a) is shown in Fig. 12(a). The maximum temperature at the inner part of the target is of the order of $10000 \mathrm{~K}$ and the radius of the heated zone is about $5 \mathrm{~mm}$. The temperature profiles along the axis at different times are plotted in Fig. 12(b). The curve labeled with $1 \mu \mathrm{s}$ shows a maximum temperature of around $3000 \mathrm{~K}$ and there exists a flat part on the right-hand side of the curve which represents the melting region. As more and more energy is deposited, the temperature rapidly increases while the flat part of the curve continuously moves towards the right. This shows that a melting wave propagates along with the beam which penetrates deeper into the target in the longitudinal direction. Because of the high temperature, the central part of the target is weakly ionized and the material is in a strongly coupled plasma state. One can therefore also study this interesting field of science in the HiRadMat experiments. From the machine protection point of view, however, the target is severely damaged. It is also seen that, due to the phenomenon of hydrodynamic tunneling, the temperature profiles become more flat with time.
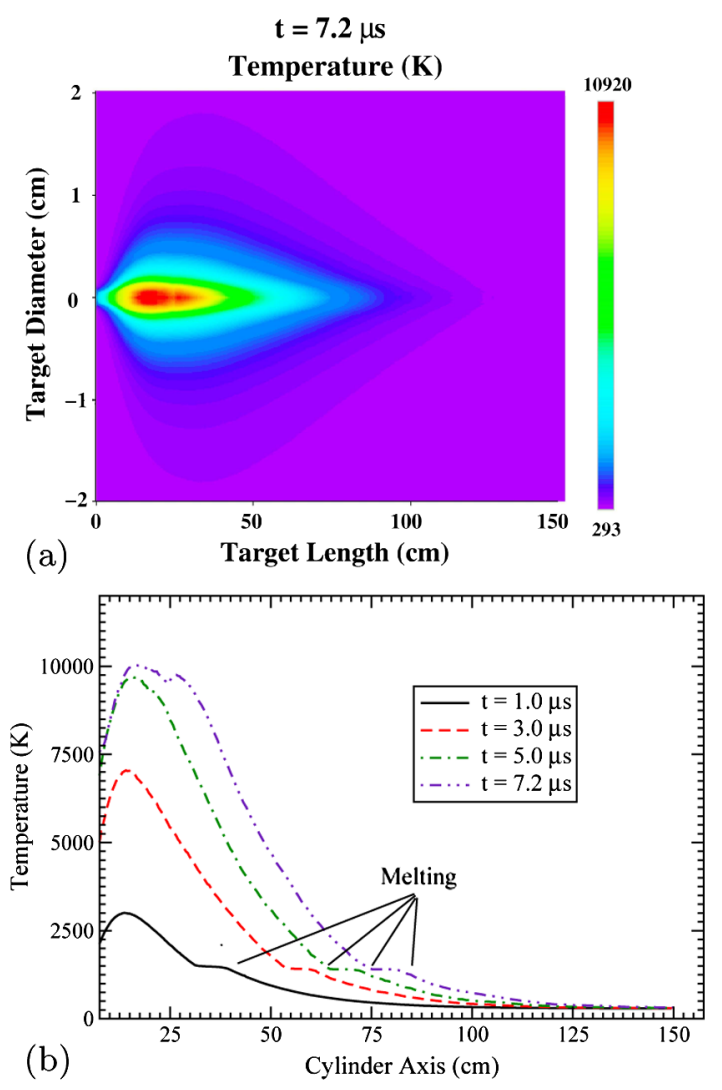

FIG. 12. (a) Temperature distribution in the cylindrical copper target, $r=5 \mathrm{~cm}, L=1.5 \mathrm{~m}$, irradiated by the $440 \mathrm{GeV}$ SPS proton beam with $\sigma=0.5 \mathrm{~mm}$ at $t=7.2 \mu \mathrm{s}$ (end of the beam) and (b) temperature profiles along the axis at different times 
The pressure distribution in the target corresponding to Fig. 11(a) is shown in Fig. 13(a). The maximum pressure at $7.2 \mu \mathrm{s}$ is around $2.28 \mathrm{GPa}$ and the radial propagation of the compression wave is clearly seen. The pressure profiles along the axis at different times are plotted in Fig. 13(b). The behavior of the pressure profiles is opposite to that of the specific energy deposition and the temperature profiles, as the maximum value of the pressure at the axis rapidly decreases with time. This is because, during the initial phase of heating, the material around the target axis still has solid density and one achieves high pressure. However, due to the radial outgoing compression wave, the density in the axial region is depleted and therefore the pressure decreases.

The density distribution in the target corresponding to Fig. 11(a) is shown in Fig. 14(a). The density in the central part of the target has been reduced to $1.18 \mathrm{~g} / \mathrm{cm}^{3}$, which is about $10 \%$ of the solid copper density. The temperature in this region is about $10000 \mathrm{~K}$ which corresponds to the physical conditions typical of strongly coupled plasmas. The density profiles along the axis at different times are plotted in Fig. 14(b). The density depletion front moves towards the right with an approximately constant speed.
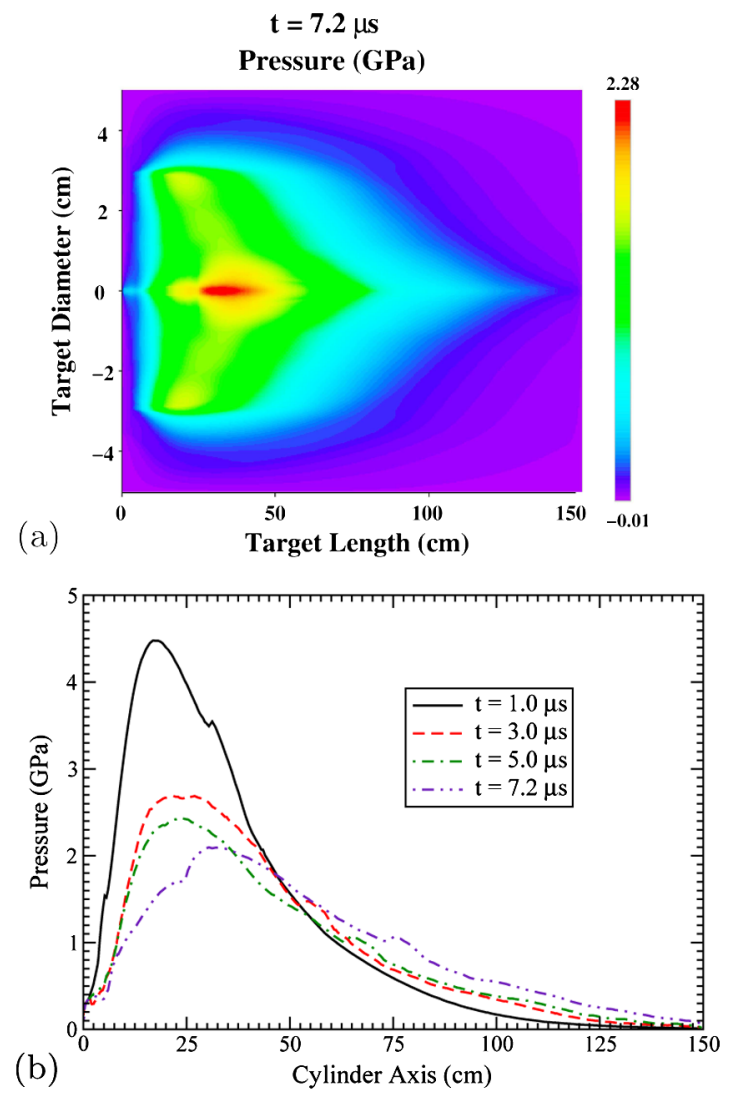

FIG. 13. (a) Pressure distribution in the cylindrical copper target, $r=5 \mathrm{~cm}, L=1.5 \mathrm{~m}$, irradiated by the $440 \mathrm{GeV}$ SPS proton beam with $\sigma=0.5 \mathrm{~mm}$ at $t=7.2 \mu \mathrm{s}$ (end of the beam) and (b) pressure profiles along the axis at different times.
The long term evolution of the pressure in the target needs to be calculated to check that the pressure waves do not get amplified as a result of interference between the waves reflected from the opposite sides of the cylinder surface. This is important for the stability of the target during the experiment.

For this purpose we present in Figs. 15(a)-15(c) the pressure distribution in the target at $t=12,14$, and $20 \mu \mathrm{s}$, respectively. At $t=7.2 \mu \mathrm{s}$, the beam is switched off and the propagation of the pressure wave in the longitudinal direction stops. However, the wave continues to propagate in the radial direction. This is seen in Fig. 15(a), where the pressure wave has just arrived at the cylinder surface and the pressure has a value $=0.8 \mathrm{GPa}$. Moreover, a V-shaped region of negative pressure has been created due to the stretching of the material radially outwards. The pressure wave reflects at the boundary and an outward velocity is imparted onto the surface.

The tensile strength of the material tends to restore the target boundary back to its original position. This is seen in Fig. 15(b) as the appearance of the negative pressure close to the boundary shows the tensile force acting in that region. The value of pressure in the reflected wave has
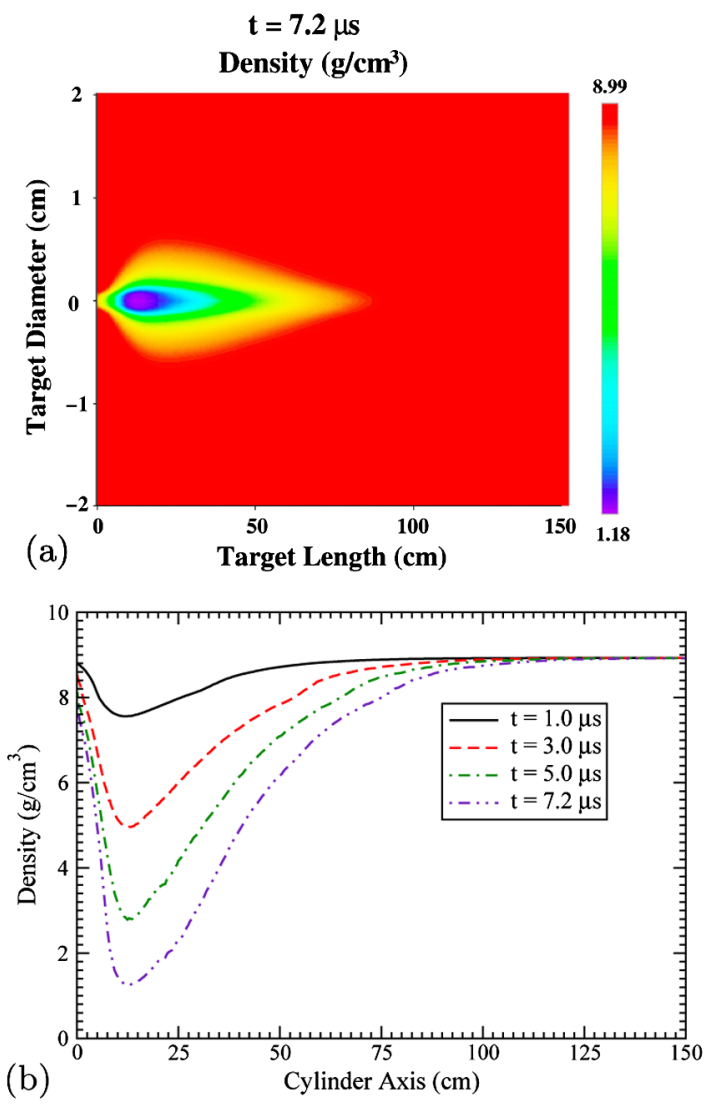

FIG. 14. (a) Density distribution in the cylindrical copper target, $r=5 \mathrm{~cm}, L=1.5 \mathrm{~m}$, irradiated by the $440 \mathrm{GeV}$ SPS proton beam with $\sigma=0.5 \mathrm{~mm}$ at $t=7.2 \mu \mathrm{s}$ (end of the beam) and (b) density profiles along the axis at different times. 


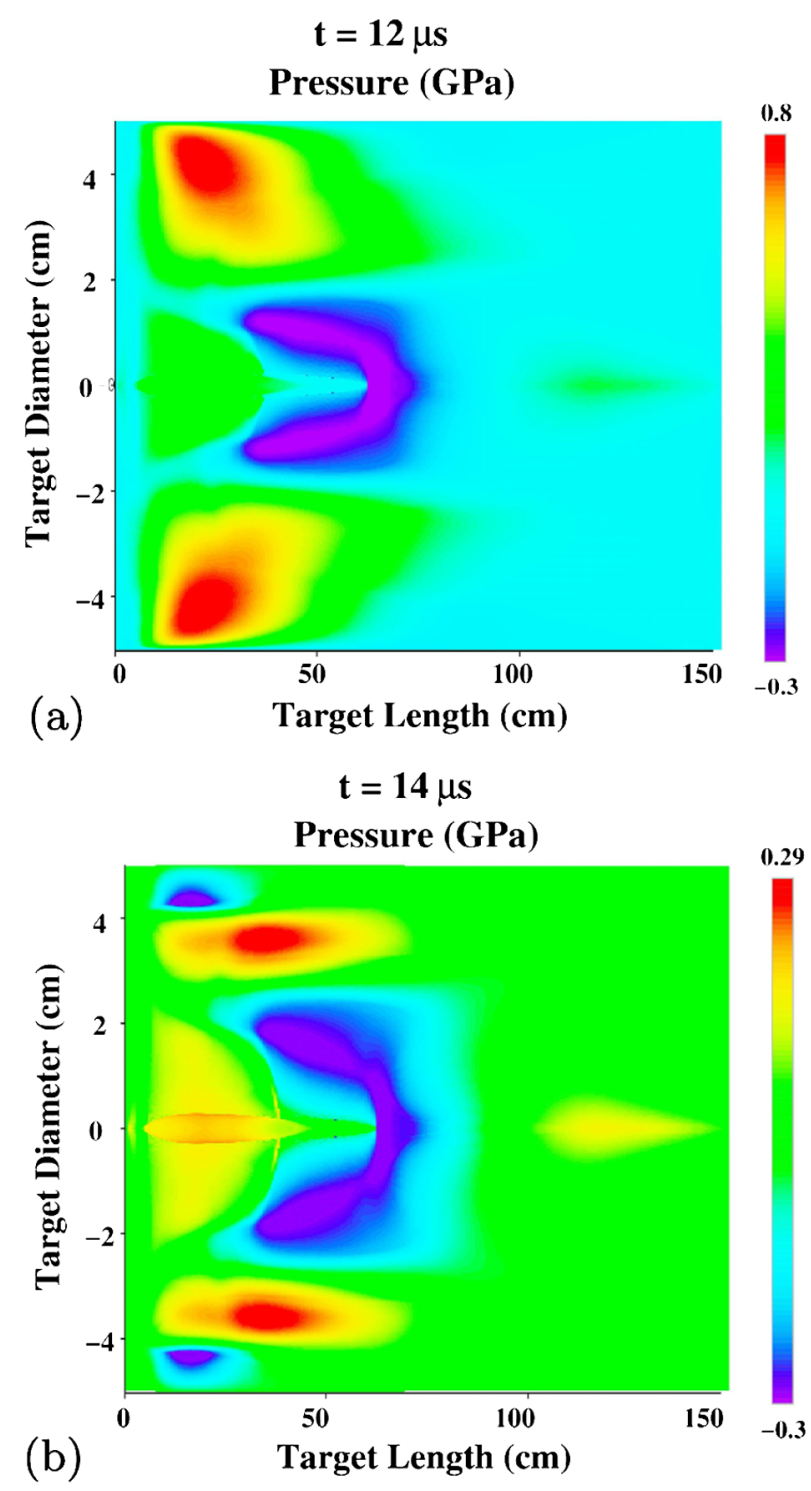

been reduced to $0.29 \mathrm{GPa}$. Figure 15(c) shows that at $t=$ $20 \mu \mathrm{s}$ the inner and the outer negative pressure zones combine and a single extended tensile region with negative pressure has been formed. The value of the negative pressure remains around- $0.3 \mathrm{GPa}$ in the calculations. It is therefore concluded that the outer shell of the target will remain intact during the experiments as the pressure waves do not amplify.

The profiles of the surface velocity at different times are plotted in Fig. 16(a). The shapes of these profiles are in accordance with that of the energy deposition profile along the axis. At $t=10 \mu \mathrm{s}$, the maximum value of the surface velocity is about $10 \mathrm{~m} / \mathrm{s}$ which increases to $18 \mathrm{~m} / \mathrm{s}$ at $t=$ $11 \mu \mathrm{s}$. The maximum value of the surface velocity increases to around $50 \mathrm{~m} / \mathrm{s}$ at $t=12 \mu \mathrm{s}$ and achieves a peak value of $58 \mathrm{~m} / \mathrm{s}$ at $t=13 \mu \mathrm{s}$. The curves plotted at later times show that the surface velocity steadily decreases. At $t=17 \mu \mathrm{s}$ it has dropped to a value of $37 \mathrm{~m} / \mathrm{s}$. This behavior of the surface velocity suggests that, if the calculations are continued for a much longer time, the surface velocity may become negative and the
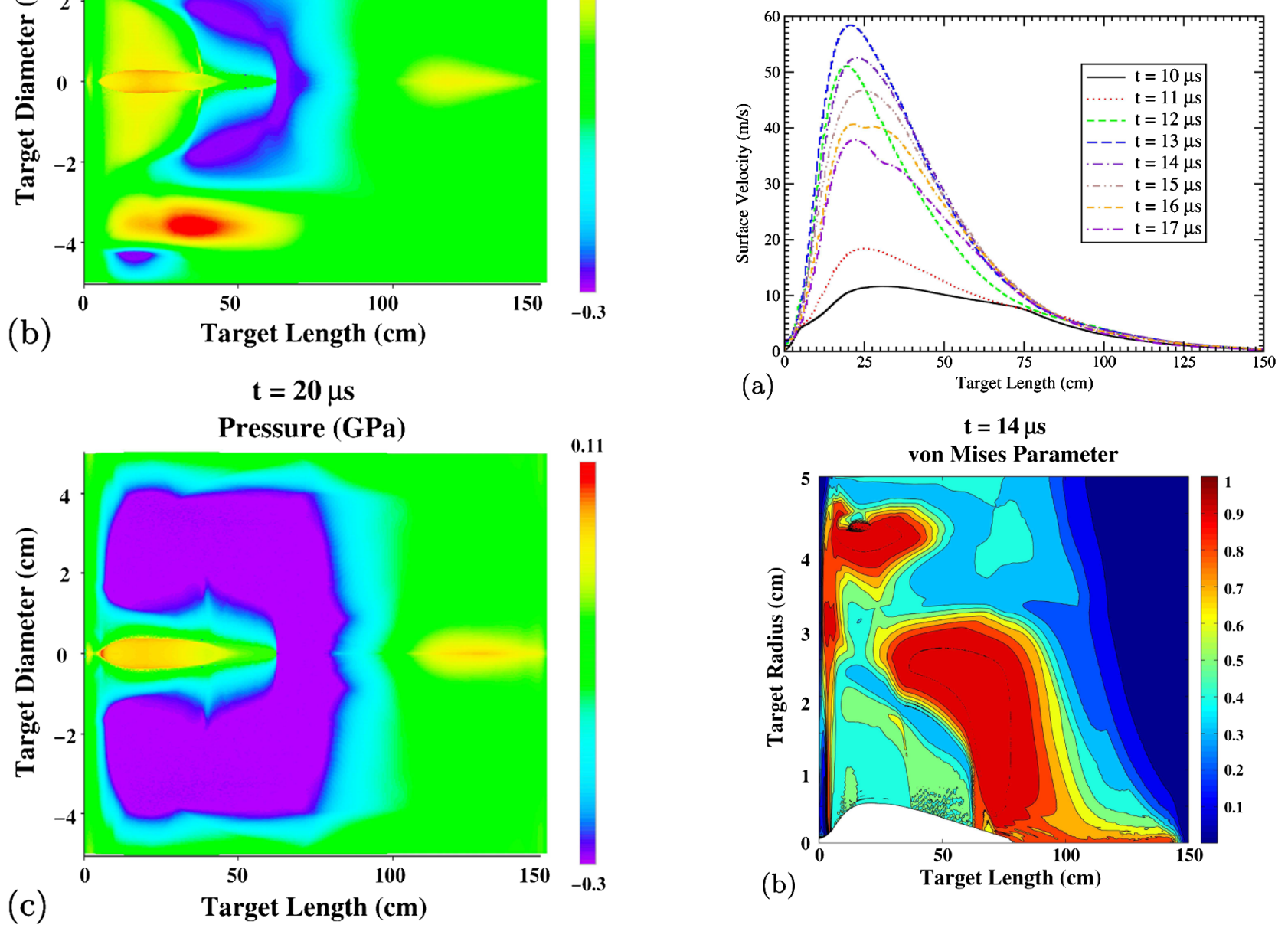

FIG. 15. Pressure distribution corresponding to Fig. 13(a); (a) at $t=12 \mu \mathrm{s}$; (b) at $t=14 \mu \mathrm{s}$; and (c) at $t=20 \mu \mathrm{s}$.

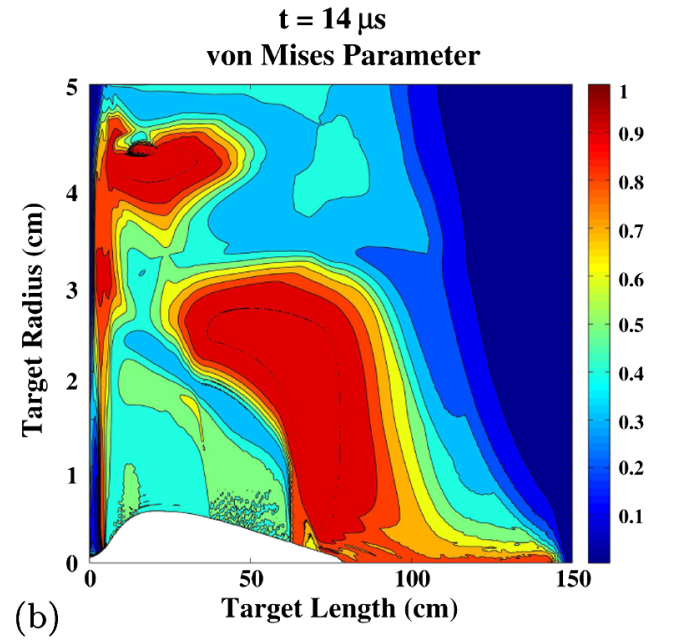

FIG. 16. (a) Profiles of the surface velocity at different times and (b) distribution of the von Mises parameter in the copper target at $t=14 \mu \mathrm{s}$. 


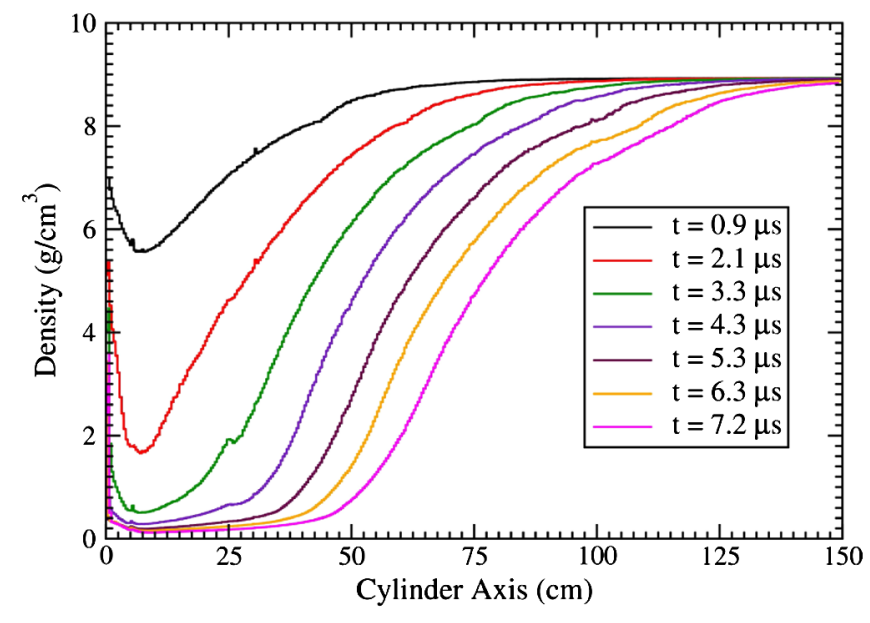

FIG. 17. Density profiles along the axis at different times in the cylindrical copper target, $r=5 \mathrm{~cm}, L=1.5 \mathrm{~m}$, irradiated by the $440 \mathrm{GeV}$ SPS proton beam with $\sigma=0.1 \mathrm{~mm}$.

surface may oscillate about its equilibrium position. This indicates that the material remains in an elastic state.

In order to check this effect in a quantitative manner, we present in Fig. 16(b) the distribution of the von Mises parameter in the solid part of the target at $t=14 \mu \mathrm{s}$. The value of the von Mises parameter is less than one in the solid part of the target, which means that the material is in an elastic state that points out towards the stability of the target during the course of the experiment.

We also briefly report the simulation results of the interaction of the SPS beam with solid copper target which has a much smaller focal spot, characterized with $\sigma=$ $0.1 \mathrm{~mm}$. Since the specific energy deposition in this case is much higher, the process of density depletion occurs at a much faster rate. We therefore used a much smaller iteration step as compared to the case of the beam with $\sigma=$ $0.5 \mathrm{~mm}$. Until $t=3.3 \mu \mathrm{s}$, the iteration step was $300 \mathrm{~ns}$ and afterwards $500 \mathrm{~ns}$. The density profiles along the target axis at different times during the irradiation are plotted in Fig. 17. It is seen that, compared to Fig. 14(a), the beam penetration in this case is significantly longer and occurs at a faster rate while the minimum value of the density along the axis is about less than $0.1 \mathrm{~g} / \mathrm{cm}^{3}$. The beam practically penetrates through the entire length of $1.5 \mathrm{~m}$ of the target.

\section{CONCLUSIONS}

In this paper we report detailed numerical simulations of the impact of the full LHC beam on a solid carbon target. The simulations have been done in a self-consistent manner by using a code for particle matter interactions, FLUKA, and the hydrodynamic code, BIG2, iteratively.

First, the FLUKA code is employed to calculate the energy deposition of the $7 \mathrm{TeV}$ LHC protons in solid carbon and this data is used as input to the BIG2 code, which calculates the thermodynamic and the hydrodynamic effects in the target material. The BIG2 code is allowed to run until the density along the target axis decreases by $15 \%-20 \%$ due to the hydrodynamics. This density distribution is used in the FLUKA code to evaluate the modified energy deposition distribution that is again used in the BIG2 for the next iteration.

A typical iteration interval of $2.5 \mu \mathrm{s}$ has been used in these calculations. Our simulations have shown that the full LHC beam will penetrate up to $25 \mathrm{~m}$ through a solid carbon target, whereas the range of a single bunch and the generated shower is around $3 \mathrm{~m}$. It is therefore very important to include the hydrodynamic effects in such calculations. This study has shown that the length of a $6 \mathrm{~m}$ long carbon absorber is not sufficient to stop the LHC beam in case of a failure of the beam dumping system. The beam is attenuated after passing through the beam stopper and is thus expected to cause less damage to the equipment. The installation of an absorber with a length of $25 \mathrm{~m}$ would be difficult and only prevent damage in a very specific failure case. Since this type of failure of the beam dump kicker magnet is highly unlikely, an installation of a $25 \mathrm{~m}$ long block has been discarded.

Experimental verification of the simulations is necessary in order to have confidence in the predicted results. For this purpose, an experiment at the HiRadMat facility is proposed. HiRadMat has been constructed at CERN to perform such experiments using the $440 \mathrm{GeV}$ SPS proton beam. To help the designing of suitable targets for these experiments, we have carried out simulations of the interaction of the SPS protons with solid copper cylindrical targets for three different values of the beam spot size $(\sigma=$ $0.1,0.2$, and $0.5 \mathrm{~mm}$ ).

The study has also shown that the beam penetration along the target axis is always present, but much more pronounced for the smaller focal spot sizes because of the higher energy density.

For $\sigma=0.5 \mathrm{~mm}$ it has been found that the pressure waves do not amplify, but become weaker after the end of beam impact. One therefore can infer that, on a much longer time scale (of the order of ms), the pressure waves may be completely damped out, which implies that the target will remain stable during the experiment.

Considering surface velocity and maximum displacement of the surface of about $500 \mu \mathrm{m}$, it can be assumed that the target material will remain in an elastic phase.

Experimental confirmation of these results will provide validation of the simulation methods and we will gain confidence in the results for the LHC.

It has been found that the target material in the beam heated region, both in the case of the LHC and the SPS, is in a state of HED matter [14-25] including WDM and strongly coupled plasma states. This would be the first experiment that generates HED matter with a high energy, high intensity proton beam. It could therefore be considered that these two unique and impressive machines, SPS and LHC, can be used as a tool to study HED physics. 


\section{ACKNOWLEDGMENTS}

The authors wish to thank G. I. Kerley for providing the multiphase, multicomponent EOS data for carbon and I. V Lomonosov for making available the semiempirical EOS data for copper. The authors also wish to thank V. Kim and S. Udrea for useful discussions. Financial support for this work was provided by the DFG, the EuCARD: ColMat, Grant Agreement No. 227579 and the Ministerio de Ciencia e Innovacin of Spain (ENE2009-09276).

[1] N. A. Tahir, B. Goddard, V. Kain, R. Schmidt, A. Shutov, I. V. Lomonosov, A. R. Piriz, M. Temporal, D. H. H. Hoffmann, and V.E. Fortov, J. Appl. Phys. 97, 083532 (2005).

[2] V. E. Fortov, B. Goel, C. D. Munz, A. L. Ni, A. Shutov, and O. V. Vorobiev, Nucl. Sci. Eng. 123, 169 (1996).

[3] A. Fasso et al., technical report, CERN, 2005.

[4] A. Fasso et al., in Conference on Computing in High Energy and Nuclear Physics, La Jolla, CA, 2003.

[5] N. A. Tahir, V. Kain, R. Schmidt, A. Shutov, I. V. Lomonosov, V. Gryaznov, A.R. Piriz, M. Temporal, D. H. H. Hoffmann, and V.E. Fortov, Phys. Rev. Lett. 94, 135004 (2005).

[6] N. A. Tahir, R. Schmidt, A. Shutov, I. V. Lomonosov, A. R. Piriz, D. H. H. Hoffmann, C. Deutsch, and V.E. Fortov, Phys. Rev. E 79, 046410 (2009).

[7] R. Schmidt, R. Assmann, E. Carlier, B. Dehning, R. Denz, B. Goddard, E. B. Holzer, V. Kain, B. Puccio, B. Todd et al., New J. Phys. 8, 290 (2006).

[8] J. Uythoven et al., in Proceedings of the 9th European Particle Accelerator Conference, Lucerne, 2004 (EPSAG, Lucerne, 2004).

[9] R. B. Appleby, B. Goddard, A. Gomez-Alonso, V. Kain, T. Kramer, D. Macina, R. Schmidt, and J. Wenninger, Phys. Rev. ST Accel. Beams 13, 061002 (2010).

[10] The LHC Design Report, CERN, 2004.

[11] C. Hessler et al., in Proceedings of IPAC 2011, San Sebastian, Spain (2011).

[12] J. Blanco Sancho, N. Conan, K. Cornelis, B. Goddard, C. Hessler, L. Jensen, V. Kain, M. Meddahi, C. Theis, P. Vojtyla, and J. Wenninger, Report No. CERN-ATS-Note2011-126 PERF, 2011.

[13] J. Blanco Sancho, N. Conan, K. Cornelis, B. Goddard, C. Hessler, L. Jensen, V. Kain, M. Meddahi, C. Theis, H. Vincke, P. Vojtyla, and J. Wenninger, Report No. CERNATS-Note-2011-56 PERF, 2011.

[14] N. A. Tahir, D. H. H. Hoffmann, J. A. Maruhn, K.-J. Lutz, and R. Bock, Phys. Plasmas 5, 4426 (1998).

[15] N. A. Tahir, D. H. H. Hoffmann, J. A. Maruhn, P. Spiller, and R. Bock, Phys. Rev. E 60, 4715 (1999).

[16] N. A. Tahir, D. H. H. Hoffmann, A. Kozyreva, A. Shutov, J. A. Maruhn, U. Neuner, A. Tauschwitz, P. Spiller, and R. Bock, Phys. Rev. E 61, 1975 (2000).

[17] N.A. Tahir, D.H.H. Hoffmann, A. Kozyreva, A. Tauschwitz, A. Shutov, J.A. Maruhn, P. Spiller,
U. Neuner, J. Jacoby, M. Roth, R. Bock, H. Juranek, and R. Redmer, Phys. Rev. E 63, 016402 (2001).

[18] N. A. Tahir, A. Kozyreva, P. Spiller, D. H. H. Hoffmann, and A. Shutov, Phys. Rev. E 63, 036407 (2001).

[19] N. A. Tahir, R. Schmidt, M. Brugger, R. Assmann, A. Shutov, I. V. Lomonosov, V. Gryaznov, A. R. Piriz, S. Udrea, D. H. H. Hoffmann, V. E. Fortov, and C. Deutsch, Phys. Plasmas 16, 082703 (2009).

[20] N. A. Tahir, A. Shutov, A. P. Zharkov, A. R. Piriz, and Th. Stöhlker, Phys. Plasmas 18, 032704 (2011).

[21] U. Neuner, R. Bock, M. Roth, P. Spiller, C. Constantin, U. N. Funk, M. Geissel, S. Hakuli, D. H. H. Hoffmann, J. Jacoby, A. Kozereva, N. A. Tahir, S. Udrea, D. Varentsov, and A. Tauschwitz, Phys. Rev. Lett. 85, 4518 (2000).

[22] U. N. Funk, R. Bock, M. Geissel, D. H. H. Hoffmann, U. Neuner, F. B. Rosmej, M. Stetter, S. Stöwe, N. A. Tahir, and A. Tauschwitz, J. Phys. IV 10, Pr5-215 (2000).

[23] S. Udrea, V. Ternovoi, N. Shilkin, A. Fertman, V.E. Fortov, D. H. H. Hoffmann, A. Hug, M. I. Kulish, V. Mintsev, P. Ni, D. Nikolaev, N. A. Tahir, V. Turtikov, D. Varentsov, and D. Yuriev, Nucl. Instrum. Methods Phys. Res., Sect. A 577, 257 (2007).

[24] J. J. Barnard, J. Armijo, R. M. More, A. Friedman, I. Kaganovich, B. G. Logan, M. M. Marinak, G. E. Penn, A. B. Sefkow, P. Santhanam et al., Nucl. Instrum. Methods Phys. Res., Sect. A 577, 275 (2007).

[25] P. Ni, D. H. H. Hoffmann, M. Kulish, D. Nikolaev, N. A. Tahir, S. Udrea, D. Varentsov, and H. Wahl, J. Phys. IV 133, 977 (2006).

[26] N. A. Tahir, R. Schmidt, M. Brugger, I. V. Lomonosov, A. Shutov, A. R. Piriz, S. Udrea, D. H. H. Hoffmann, and C. Deutsch, Laser Part. Beams 25, 639 (2007).

[27] N. A. Tahir, R. Schmidt, M. Brugger, R. Assmann, A. Shutov, I. V. Lomonosov, V.E. Fortov, A. R. Piriz, C. Deutsch, and D.H.H. Hoffmann, Nucl. Instrum. Methods Phys. Res., Sect. A 606, 128 (2009).

[28] N. A. Tahir, R. Schmidt, M. Brugger, A. Shutov, I. V. Lomonosov, A. R. Piriz, and D. H.H. Hoffmann, Laser Part. Beams 27, 475 (2009).

[29] N. A. Tahir et al., in Proceedings of IPAC 2011, San Sebastian, Spain (2011).

[30] G. Kerley, technical report, Sandia National Lab, 2001.

[31] D. C. Wilson et al., in Proceedings of PAC 1993, San Sebastian, Spain (1993).

[32] N. A. Tahir, R. Schmidt, M. Brugger, R. Assmann, A. V. Shutov, I. V. Lomonosov, A. R. Piriz, D. H. H. Hoffmann, C. Deutsch, and V. E. Fortov, New J. Phys. 10, 073028 (2008).

[33] A. V. Bushman and V. E. Fortov, Sov. Technol. Rev. B 1, 219 (1987).

[34] I. V. Lomonosov, Laser Part. Beams 25, 567 (2007).

[35] I. V. Lomonosov and N. A. Tahir, Appl. Phys. Lett. 92, 101905 (2008).

[36] S. Myers, in Proceedings of the IPAC'10 Conference, Kyoto, Japan (ICR, Kyoto, 2010).

[37] K. Wittenburg (private communication).

[38] V. Shiltsev, in Proceedings of IPAC 2011, San Sebastian, Spain (2011).

[39] J. Galambos, in Proceedings of HB 2010, Morschach, Switzerland (2010). 